\title{
Sampled-data synchronization of chaotic Lur'e systems via an adaptive event-triggered approach
}

DOI:

10.1016/j.ins.2018.06.012

\section{Document Version}

Accepted author manuscript

Link to publication record in Manchester Research Explorer

\section{Citation for published version (APA):}

Li, T., Yuan, R., Fei, S., \& Ding, Z. (2018). Sampled-data synchronization of chaotic Lur'e systems via an adaptive event-triggered approach. Information Sciences, 462, 40-54. https://doi.org/10.1016/j.ins.2018.06.012

\section{Published in:}

Information Sciences

\section{Citing this paper}

Please note that where the full-text provided on Manchester Research Explorer is the Author Accepted Manuscript or Proof version this may differ from the final Published version. If citing, it is advised that you check and use the publisher's definitive version.

\section{General rights}

Copyright and moral rights for the publications made accessible in the Research Explorer are retained by the authors and/or other copyright owners and it is a condition of accessing publications that users recognise and abide by the legal requirements associated with these rights.

\section{Takedown policy}

If you believe that this document breaches copyright please refer to the University of Manchester's Takedown Procedures [http://man.ac.uk/04Y6Bo] or contact uml.scholarlycommunications@manchester.ac.uk providing relevant details, so we can investigate your claim.

\section{OPEN ACCESS}




\title{
Sampled-data synchronization of chaotic Lur'e systems via an adaptive event-triggered approach
}

\author{
Tao Li ${ }^{a, *}$, Ruiting Yuan ${ }^{a}$, Shumin Fei ${ }^{\text {b }}$, Zhengtao Ding ${ }^{c}$ \\ ${ }^{a}$ School of Automation Engineering, Nanjing University of Aeronautics and Astronautics, Nanjing 210016, PR China; \\ ${ }^{\mathrm{b}}$ School of Automation, Southeast University, Nanjing 210096, PR China; \\ ${ }^{\mathrm{c}}$ School of Electrical and Electronic Engineering, The University of Manchester, Manchester M13 9PL, United Kingdom.
}

\begin{abstract}
In this paper, based on nonuniform sampling, the master-slave synchronization in a class of Lur'e systems is studied via using the sampled outputs of error system. Different from existing results, the transmission of control signals is determined by a novel adaptive event-triggered scheme, where the triggering thresholds depend on the dynamic behaviors of controlled systems rather than the predetermined constants as some traditional ones. Through choosing two augmented Lyapunov-Krasovskii functionals (LKFs), some delay-dependent synchronization criteria are formulated and the conservatism can be effectively reduced owing to the utilization of Wirtinger-based inequalities and delay-product-type LKF terms. Especially, the existence of the controller can be easily checked since the derived conditions are presented via LMI forms. Finally, two numerical examples with comparisons and simulations are given to illustrate the proposed results.
\end{abstract}

Key words: Chaotic Lur'e systems; master-slave synchronization; adaptive event-triggered scheme; sampled-data control; time-varying delay

\section{Introduction}

In past decades, the research on synchronization control of various chaotic systems has received considerable attention since the pioneering works of Pecora and Carroll were reported $[1,20]$. The works show that as some conditions are satisfied, a chaotic system (the slave system) may become synchronized to another identical one (the master system) if the master system sends some driving signals to the slave one. Presently, it is widely known that there exist many benefits of chaos synchronization in various engineering applications, such as teleoperation control, secure communication, and image processing. It is worth noting that the dynamical behaviors of chaotic systems are highly sensitive to small changes of initial values and the trajectories in phase space are bounded due to the presence of nonlinearity. Therefore, the problem on chaos synchronization has been widely investigated and a large number of elegant results have been reported [2,3,6,8,10,11,13,14,23-26,28,32,36-40].

Meanwhile, Lur'e system is an important theoretic model describing how nonlinear uncertainties reflect the dynamic behaviors in a system, which can include linear models and nonlinear ones as its special case and exhibit some chaotic behaviors [18,31]. Therefore, the synchronization in Lur'e systems has received considerable attention and many interesting results have been proposed [2,3,6,8,13,14,23-26,32,36-39]. The Ref. [6] studied two nonlinear Lur'e ones subject to control saturation and assumed that their outputs were measurable. In [36], two Lur'e networks of homogeneous dimensions were analyzed to tackle the master-slave synchronization only when the relative measurements were available. It is worth noting that time-delay is an inherent feature in physical processes and may lead to instability or significantly deteriorate system performance. Thus some works have tackled the time-delay Lur'e

\footnotetext{
ऋ Corresponding author at: School of Automation Engineering, Nanjing University of Aeronautics and Astronautics, Nanjing 210016, PR China. Tel.: +86 2584892301.
}

Email address: autolitao@nuaa.edu.cn (Tao Li). 
systems $[3,23,24,37,39]$. In [3], an impulsive controller involving time-delay was designed to study the exponential synchronization and in $[23,24,37,39]$, some easy-to-test criteria were presented.

In practical situations, since digital controllers allow for the task only by using the error outputs of the master system and slave one at discrete time instants, they are more preferable than the continuous-time ones and widely utilized to study the synchronization $[2,8,13,14,24-26,32,37-39]$. Especially, in [2], the $H_{\infty}$ synchronization was studied and the Ref. [13] put forward a novel discontinuous LKF term to reduce the conservatism and further gave comparisons with existing ones. The work [14] considered the networked circumstances and the Refs. [24,25,28] utilized some Wirtinger-based inequalities to extend the application areas, in which stochastic disturbance and non-fragility were involved. Yet the Lur'e systems in $[13,14,25,28]$ did not include time-delay, which was considered in $[2,8,24,26,37,39]$. It should be pointed out that the controllers in $[2,8,13,14,24-26,37-39]$ were time-triggered and a maximum sampling interval had to be carefully selected. This type of triggered scheme unavoidably leads to unnecessary utilization of communication bandwidth. In order to overcome this point, a novel idea described as event-triggered technique was put forward. Compared with time-triggered scheme, the event-triggered one is more efficient and typically requires less data transmissions since the sampler is triggered only when the value of the designed function exceeds a given threshold value. Therefore, this scheme has been widely employed [4,7,14,28,32,33,40,42-45], which was also applied to tackle the synchronization issue of many systems including Lur'e ones $[14,28,32,40]$.

Though the results above are elegant, there still exist two challenges to be addressed. Firstly, since the eventtriggered technique based on sampled data was put forward to moderate the transmission burden [35], it has received numerous improvements $[4,14,28,32,33,40,42-45]$. Yet, most triggering thresholds were pre-selected constants belonging to $(0,1]$ and could not adapt to the real-time performance of controlled system. Though the work [7] has given preliminary discussions on on-line optimization algorithms of achieving the desired thresholds. However, its adaptive condition was presented via a simple form and could not reflect the whole information. Especially, as we know, no works have employed the adaptive event-triggered technique to tackle the synchronization issue. Secondly, as for LKF candidate, a novel discontinuous LKF term based on free-weighting matrices was put forward in [13] and some comparisons were also given with the one based on Wirtinger's inequalities [16]. Yet, few works have not combined the LKF terms in $[13,16]$ to extend the application areas. Furthermore, the derivative of LKF term in [13] results in one Zero term, which will unavoidably induce the conservatism. Especially, most recently, the Refs. [18,46] put forward some novel delay-product-type LKF terms, which can reduce the conservatism more effectively than ever. Yet no present works have utilized this technique to tackle the synchronization in Lur'e systems. It is worth noting that, as for time-delay Lur'e systems based sampling control, there always exist two types of time-delays, i.e., one is embedded in system and the other is a modeled one $[2,8,24,26,37,39]$. However, few works have utilized their interconnection to construct the LKF. Though the work [5] has studied this point on time-delay neutral system, the LKF terms seemed to be simple and multiple integral ones were not utilized. To the best knowledge of authors, the research on adaptive event-triggered synchronization in master-slave Lur'e systems has not appeared with the aid of latest relaxed integral inequalities and LKF terms, which motivates this presented work.

In this paper, based on sampled data, the master-slave synchronization in Lur'e systems with time-varying delay will be deeply studied and an adaptive event-triggered scheme is designed to improve the transmission efficiency. Together with the information on time-delays and nonlinear functions, two augmented LKFs will be constructed. By employing some Wirtinger-based inequalities and convex combination technique, two novel conditions will present less conservatism and easily checked owing to that they are presented in terms of the LMIs. Finally, the efficiency of the derived criteria will be demonstrated by resorting to two numerical examples.

Notations The term LKF is the abbreviation of Lyapunov-Krasovskii functional and $\mathbf{N}$ represent the set of natural numbers; the set $\mathbf{R}^{n}$ denotes the $n$-dimensional Euclidean space and $\mathbf{R}^{n \times m}$ is the set of $n \times m$ real matrices; $I_{n}$ denotes the $n \times n$ identity matrix; $\operatorname{sym}\{X\}$ means $\operatorname{sym}\{X\}=X+X^{T} ; A \otimes B$ denotes the Kronecker product of $A, B$, and the symmetric term in a symmetric matrix is denoted by $*$.

\section{Problem formulations and lemmas}

In this paper, we consider the time-delay Lur'e systems of master-slave case described as

$$
\begin{aligned}
& \mathcal{M}:\left\{\begin{array}{l}
\dot{x}(t)=A x(t)+B x(t-\tau(t))+D h(E x(t)), \\
p(t)=F x(t),
\end{array}\right. \\
& \mathcal{S}:\left\{\begin{array}{l}
\dot{z}(t)=A z(t)+B z(t-\tau(t))+D h(E z(t))+u(t), \\
q(t)=F z(t),
\end{array}\right.
\end{aligned}
$$


where $\mathcal{M}$ is the master system and $\mathcal{S}$ is the slave system; here $x(t), z(t) \in \mathbf{R}^{n}$ are the state vectors and the output ones are presented as $p(t), q(t) \in \mathbf{R}^{n_{0}} ; A=\left[a_{i j}\right]_{n \times n}, B=\left[b_{i j}\right]_{n \times n}, F=\left[f_{i j}\right]_{n_{0} \times n}, E=\left[E_{1}^{T}, E_{2}^{T}, \cdots, E_{n_{1}}^{T}\right]^{T}$ are constant matrices; moreover $h(\cdot)=\left[h_{1}(\cdot), h_{2}(\cdot), \cdots, h_{n_{1}}(\cdot)\right]^{T}$ denotes the nonlinear vector-valued function which satisfies the global Lipschitz condition.

The following assumptions will be utilized throughout this paper.

H1. The function $\tau(t)$ denotes the time-varying delay satisfying

$$
0 \leq \tau(t) \leq \bar{\tau}, \quad \mu_{0} \leq \dot{\tau}(t) \leq \mu_{1},
$$

where $\bar{\tau}, \mu_{0}, \mu_{1}$ are constant scalars.

H2. There exist the constants $\sigma_{i}^{+}, \sigma_{i}^{-}\left(i=1, \cdots, n_{1}\right)$ such that the nonlinear function $h_{i}(\cdot)$ in $(1)-(2)$ satisfies

$$
\left[h_{i}(\xi)-\sigma_{i}^{+} \xi\right]\left[h_{i}(\xi)-\sigma_{i}^{-} \xi\right] \leq 0, \forall \xi \in \mathbf{R} .
$$

Here, we introduce $\bar{\Sigma}=\operatorname{diag}\left(\sigma_{1}^{+}, \cdots, \sigma_{n_{1}}^{+}\right), \Sigma=\operatorname{diag}\left(\sigma_{1}^{-}, \cdots, \sigma_{n_{1}}^{-}\right)$, and

$$
\Sigma_{1}=\operatorname{diag}\left(\sigma_{1}^{+} \sigma_{1}^{-}, \cdots, \sigma_{n_{1}}^{+} \sigma_{n_{1}}^{-}\right), \Sigma_{2}=\operatorname{diag}\left(\frac{\sigma_{1}^{+}+\sigma_{1}^{-}}{2}, \cdots, \frac{\sigma_{n_{1}}^{+}+\sigma_{n_{1}}^{-}}{2}\right) .
$$

Now by defining $\varepsilon(t)=z(t)-x(t)$, the error system together with the controller can be given as

$$
\dot{\varepsilon}(t)=A \varepsilon(t)+B \varepsilon(t-\tau(t))+D f(E \varepsilon(t))+u(t),
$$

where $f(E \varepsilon(t))=h(E z(t))-h(E x(t))$. Based on Ref. [27], one can check that $f_{i}(\cdot)\left(i=1,2, \cdots, n_{1}\right)$ satisfies H2.

In this work, we will study the synchronization task via sampled-data feedback. Since only discrete-time measurements of $p(t), q(t)$ can be used for the control purpose, we can have the measurements $p\left(t_{l}\right), q\left(t_{l}\right)(l \in \mathbf{N})$ at the sampling instant $t_{l}$. In traditional control, it is always assumed that the control input is generated by using a zero-order-hold $(\mathrm{ZOH})$ function with a sequence of holding time $t \in\left[t_{l}, t_{l+1}\right)$, where $t_{l}$ is the transmitted instant from sensor to controller. Yet, in practice, the sampled-data packets transmitted through a communication channel usually suffer some unfavorable factors such as transmission delay. Similar to [44], we take the transmission delay as a constant $\bar{d}$ and the property of the $\mathrm{ZOH}$ altogether into account, the design controller can be presented as

$$
u(t)=K\left[q\left(t_{l}\right)-p\left(t_{l}\right)\right], \quad t \in \mathbf{\Omega}_{l} \doteq\left[t_{l}+\bar{d}, t_{l+1}+\bar{d}\right),
$$

where $K$ is the controller gain waiting to be designed. Now as for the controller in (6), normally, in a conventional time-triggered scheme, there always assumes that the sampling intervals are identical in all time, that is, $t_{l+1}=t_{l}+h$ for a constant sampling period $h>0$. Yet, in communication networks, since limited bandwidth, loading changes, device faults, outer disturbance, and other unknown factors are always existent, the sampling period has to be time variable and then the assumption above will be relaxed. That is to say, in this work, the sampling period $h_{i}(i \in \mathbf{N})$ is allowed to be variable and belongs to a given interval, i.e., $h_{i} \in[\underline{h}, \tilde{h}]$ with $\underline{h}>0$. Meanwhile, since time-triggered scheme unavoidably leads to unnecessary utilization of communication network, then an adaptive event-triggered one will be designed in this work. That is to say, an event for system (5) is triggered as soon as the following relationship is violated $\mathcal{E}(\cdot, \cdot, \cdot) \leq 0$. Here the event-trigged scheme $\mathcal{E}(\cdot, \cdot, \cdot)$ can be described as

$$
\mathcal{E}\left(e\left(i_{s}\right), \varepsilon\left(t_{l}\right), \varepsilon\left(i_{s}\right)\right)=e^{T}\left(i_{s}\right) \Phi_{1} e\left(i_{s}\right)-\alpha(t) \varepsilon^{T}\left(t_{l}\right) \Phi_{2} \varepsilon\left(t_{l}\right)-\beta(t) \varepsilon^{T}\left(i_{s}\right) \Phi_{2} \varepsilon\left(i_{s}\right)
$$

where $i_{s}=t_{l}+\sum_{i=1}^{s-1} h_{i}$ is the current sampling instant, $t_{l}$ is the latest transmitted one, $e\left(i_{s}\right)=\varepsilon\left(i_{s}\right)-\varepsilon\left(t_{l}\right)$, and $\Phi_{i}>0(i=1,2)$ are the weight matrices. Especially, the functions $\alpha(t), \beta(t)$ satisfy

$$
\begin{aligned}
& \dot{\alpha}(t)=\frac{1}{\alpha(t)}\left[\frac{1}{\alpha(t)}-\delta_{1}\right]\left[e^{T}\left(i_{s}\right) \Phi_{1} e\left(i_{s}\right)-\theta_{1} \varepsilon^{T}\left(i_{s}\right) \Phi_{2} \varepsilon\left(i_{s}\right)+\theta_{1} \varepsilon^{T}\left(t_{l}\right) \Phi_{2} \varepsilon\left(t_{l}\right)\right], \\
& \dot{\beta}(t)=\frac{1}{\beta(t)}\left[\frac{1}{\beta(t)}-\delta_{2}\right]\left[e^{T}\left(i_{s}\right) \Phi_{1} e\left(i_{s}\right)-\theta_{2} \varepsilon^{T}\left(t_{l}\right) \Phi_{2} \varepsilon\left(t_{l}\right)+\theta_{2} \varepsilon^{T}\left(i_{s}\right) \Phi_{2} \varepsilon\left(i_{s}\right)\right],
\end{aligned}
$$


where $\delta_{i}, \theta_{i}(i=1,2)$ are given positive constants such that $\frac{1}{\delta_{1}} \leq \alpha(t) \leq \theta_{2}, \frac{1}{\delta_{2}} \leq \beta(t) \leq \theta_{1}$.

Remark 1. Based on the strategy (7), one can check that, firstly, our scheme does not only include the information on triggering error, but also depends on the latest updated released data and current sampling one; secondly, the triggering thresholds $\alpha(t), \beta(t)$ are not predetermined constants but two time-varying functions regulated by the adaptive laws in (8)-(9), in which the triggering error, the previously transmitted data, and current one are fully studied. Thus, our scheme can include the one in [7] as its special case. Especially, since $\Phi_{i}(i=1,2)$ are different, then the restricted condition $0<\alpha(t)<1$ in [7] is no longer needed.

Remark 2. Together with the event-triggered scheme described in (7)-(9), firstly, once the systems (1)-(2) achieve the desired synchronization, then $\dot{\alpha}(t)=\dot{\beta}(t)=0$, which means that the triggering thresholds $\alpha(t), \beta(t)$ do not need regulation any more; secondly, though $\delta_{i}>0, \theta_{i}>0(i=1,2)$ are any given constants, the condition $\frac{1}{\delta_{1}} \leq \theta_{2}, \frac{1}{\delta_{2}} \leq \theta_{1}$ will further offer an effective technique how to choose those scalars before the computing; thirdly, once $\alpha(t), \beta(t)$ converge to be constants, the event-triggered scheme in (7) can be reduced to the traditional case $[4,14,28,32,33,40,42-$ 45]. Overall, our scheme can present more generalities.

Based on the triggered scheme (7)-(9), it is well known that there are some discarded data between two transmitted ones. For introducing the proposed scheme at every sampling instant, the current sampled data is determined whether it should be transmitted or not. Then similar to the Ref. [35], we also divide the holding interval $\boldsymbol{\Omega}_{l}$ of the ZOH into subsets $\boldsymbol{\Xi}_{s} \doteq\left[i_{s}+\bar{d}, i_{s}+h_{s+1}+\bar{d}\right)$, i.e., $\boldsymbol{\Omega}_{l}=\cup \boldsymbol{\Xi}_{s}\left(s=0,1, \cdots, t_{l+1}-t_{l}-1\right)$.

Now as for $t \in \boldsymbol{\Xi}_{s}$, if we denote $\eta(t)=t-i_{s}$, the designed controller in (6) can be rewritten as

$$
u(t)=K F \varepsilon\left(t_{l}\right)=K F\left[\varepsilon\left(t_{l}\right)-\varepsilon\left(i_{s}\right)+\varepsilon\left(i_{s}\right)\right]=K F\left[\varepsilon(t-\eta(t))-e\left(i_{s}\right)\right],
$$

where $\dot{\eta}(t)=1$ and $0 \leq \bar{d} \leq \eta(t) \leq h_{s+1}+\bar{d} \leq \tilde{h}+\bar{d} \doteq \bar{h}$. Then the system (5) can be equivalently converted to

$$
\dot{\varepsilon}(t)=A \varepsilon(t)+B \varepsilon(t-\tau(t))+D f(E \varepsilon(t))+K F \varepsilon(t-\eta(t))-K F e\left(i_{s}\right), t \in \mathbf{\Xi}_{s} .
$$

In what is next, some essential lemmas are introduced.

Lemma 1. (Extended reciprocal convex lemma; [41]) For $\alpha \in(0,1)$, let $R_{1}, R_{2} \in \mathbf{R}^{n \times n}$ be real symmetric positive matrices and the vectors $\omega_{1}, \omega_{2} \in \mathbf{R}^{n}$. Thus for any $Y_{1}, Y_{2} \in \mathbf{R}^{n \times n}$ and $\mathcal{F}(\alpha)=\frac{1}{\alpha} \omega_{1}^{T} R_{1} \omega_{1}+\frac{1}{1-\alpha} \omega_{2}^{T} R_{2} \omega_{2}$, then

$$
\mathcal{F}(\alpha) \geq\left[\begin{array}{l}
\omega_{1} \\
\omega_{2}
\end{array}\right]^{T}\left\{\left[\begin{array}{cc}
R_{1} & \alpha Y_{1}+(1-\alpha) Y_{2} \\
* & R_{2}
\end{array}\right]+\left[\begin{array}{cc}
(1-\alpha)\left(R_{1}-Y_{1} R_{2}^{-1} Y_{1}^{T}\right) & 0 \\
* & \alpha\left(R_{2}-Y_{2} R_{1}^{-1} Y_{2}^{T}\right)
\end{array}\right]\right\}\left[\begin{array}{l}
\omega_{1} \\
\omega_{2}
\end{array}\right]
$$

Lemma 2.(Integral inequality; [21]) For any constant matrix $M>0$, the following inequalities hold for all continuously differentiable function $\varphi$ in $[a, b] \rightarrow \mathbf{R}^{n}$ :

$$
\begin{aligned}
& -(b-a) \int_{a}^{b} \varphi^{T}(s) M \varphi(s) d s \leq-\left(\int_{a}^{b} \varphi(s) d s\right)^{T} M\left(\int_{a}^{b} \varphi(s) d s\right)-3 \Theta^{T} M \Theta \\
& -\frac{b^{2}-a^{2}}{2} \int_{a}^{b} \int_{t+\theta}^{t} \varphi^{T}(s) M \varphi(s) d s d \theta \leq-\left(\int_{a}^{b} \int_{t+\theta}^{t} \varphi(s) d s d \theta\right)^{T} M\left(\int_{a}^{b} \int_{t+\theta}^{t} \varphi(s) d s d \theta\right),
\end{aligned}
$$

where $\Theta=\int_{a}^{b} \varphi(s) d s-\frac{2}{b-a} \int_{a}^{b} \int_{a}^{s} \varphi(u) d u d s$

Lemma 3. (Integral inequality; [9]) For any constant matrix $M>0$, the following inequality holds for all continuously differentiable function $\varphi$ in $[a, b] \rightarrow \mathbf{R}^{n}$ :

$$
-\frac{(b-a)^{2}}{2} \int_{a}^{b} \int_{a}^{s} \varphi^{T}(u) M \varphi(u) d u d s \leq-\left(\int_{a}^{b} \int_{a}^{s} \varphi(u) d u d s\right)^{T} M\left(\int_{a}^{b} \int_{a}^{s} \varphi(u) d u d s\right)-2 \Theta^{T} M \Theta,
$$


where $\Theta=\int_{a}^{b} \int_{a}^{s} \varphi(u) d u d s-\frac{3}{b-a} \int_{a}^{b} \int_{a}^{s} \int_{a}^{u} \varphi(v) d v d u d s$.

Lemma 4.(Integral inequality; [22]) For a vector $\omega$, real scalars $a \leq b$, symmetric matrix $R>0$ such that the integration is well defined, then the following inequality holds,

$$
-(b-a) \int_{a}^{b} \dot{\omega}^{T}(s) R \dot{\omega}(s) d s \leq-\chi_{1}^{T} R \chi_{1}-3 \chi_{2}^{T} R \chi_{2}-5 \chi_{3}^{T} R \chi_{3},
$$

where $\chi_{1}=\omega(b)-\omega(a), \chi_{2}=\omega(b)+\omega(a)-\frac{2}{b-a} \int_{a}^{b} \omega(s) d s$, and $\chi_{3}=\omega(b)-\omega(a)+\frac{6}{b-a} \int_{a}^{b} \omega(s) d s-\frac{12}{(b-a)^{2}} \int_{a}^{b} \int_{s}^{b} \omega(\theta) d \theta d s$. Lemma 5.(Convex combination technique; [17]) Suppose that $\Omega, \Xi_{i j}, \Xi_{m n}(i, m=1,2,3,4 ; j, n=1,2)$ are the constant matrices of appropriate dimensions, $\alpha \in[0,1], \beta \in[0,1], \gamma \in[0,1]$, and $\delta \in[0,1]$, then

$$
\Omega+\left[\alpha \Xi_{11}+(1-\alpha) \Xi_{12}\right]+\left[\beta \Xi_{21}+(1-\beta) \Xi_{22}\right]+\left[\gamma \Xi_{31}+(1-\gamma) \Xi_{32}\right]+\left[\delta \Xi_{41}+(1-\delta) \Xi_{42}\right]<0
$$

holds, if and only if the inequalities $\Omega+\Xi_{i j}+\Xi_{m n}<0 \quad(i, m=1,2,3,4 ; j, n=1,2)$ can be true.

\section{Mixed-delay-dependent synchronization criteria}

In order for simplification, we initially set $n_{0}=n_{1}=n$ and present some useful denotations in A Appendix. In what is next, based on the scheme in (7)-(9) and Lemmas 1-5, we will establish one sufficient condition on the controller design of the error system (11).

Theorem 1. For given scalars $\bar{\tau} \geq 0, \bar{h} \geq 0, \bar{\theta} \geq 0, \bar{\delta} \geq 0, \delta \geq 0, \delta_{i}>0, \theta_{i}>0(i=1,2), \mu_{0}, \mu_{1}, \bar{\mu}$ in (3) and (51), if there exist matrices $P>0, Q_{i}>0(i=1,2, \cdots, 6), R_{j}>0(j=1,2,3,4), S_{l}>0, \Phi_{l}>0, U_{l}(l=1,2)$, $U>0, V>0, W>0, M>0, X>0, M_{1}, N_{m}(m=1,2, \cdots, 6)$ making $\bar{R}_{l}=\operatorname{diag}\left(R_{l}, 3 R_{l}, 5 R_{l}\right),\left[\begin{array}{cc}\bar{R}_{l} & U_{l} \\ * & \bar{R}_{l}\end{array}\right] \geq 0(l=$ $1,2),\left[\begin{array}{ccc}N_{1} & N_{2} & N_{3} \\ * & N_{4} & N_{5} \\ * & * & N_{6}\end{array}\right] \geq 0$, and diagonal matrices $Q>0, R>0, H>0$ such that the LMI in (12) hold

$$
\left[\begin{array}{ccc}
\boldsymbol{\Omega}+\boldsymbol{\Upsilon}+\bar{\mu} \boldsymbol{\Phi}_{i}+\bar{h} \mathbf{\Psi}_{j}+\bar{\tau} \boldsymbol{\Theta}_{k} & \Pi_{3-k}^{T} U_{1} & \Gamma_{3-j}^{T} U_{2} \\
* & -\bar{\tau} \bar{R}_{1} & 0 \\
* & * & -\bar{h} \bar{R}_{2}
\end{array}\right]<0(i, j, k=1,2)
$$

where $\boldsymbol{\Upsilon}=\operatorname{sym}\left\{\Upsilon_{0} \boldsymbol{\Xi} \$_{1}^{T}+\Lambda P \$_{2}^{T}\right\}-\frac{1}{\bar{\tau}}\left[\begin{array}{c}\Pi_{1} \\ \Pi_{2}\end{array}\right]^{T}\left[\begin{array}{cc}\bar{R}_{1} & U_{1} \\ * & \bar{R}_{1}\end{array}\right]\left[\begin{array}{c}\Pi_{1} \\ \Pi_{2}\end{array}\right]-\frac{1}{h}\left[\begin{array}{c}\Gamma_{1} \\ \Gamma_{2}\end{array}\right]^{T}\left[\begin{array}{cc}\bar{R}_{2} & U_{2} \\ * & \bar{R}_{2}\end{array}\right]\left[\begin{array}{c}\Gamma_{1} \\ \Gamma_{2}\end{array}\right], \boldsymbol{\Phi}_{i}=e_{2}^{T} Q_{i} e_{2}$, and

$$
\begin{aligned}
& \boldsymbol{\Psi}_{j}=\operatorname{sym}\left\{\Upsilon_{j} \boldsymbol{\Xi} \$_{1}^{T}+\Phi_{j} P \$_{2}^{T}\right\}-e_{8+j}^{T} S_{2} e_{8+j}-\left(e_{8+j}-e_{12+j}\right)^{T}\left(3 S_{2}\right)\left(e_{8+j}-e_{12+j}\right)-\Gamma_{3-j}^{T} \frac{R_{2}}{\bar{h}^{2}} \Gamma_{3-j}, \\
& \boldsymbol{\Xi}=\bar{h}\left(N_{1}+\frac{\bar{h}^{2}}{3} N_{4}-\operatorname{sym}\left\{\left[N_{5} N_{5} 0\right]\right\}\right)+\operatorname{sym}\left\{\left[N_{3}-N_{3} 2 N_{5}\right]\right\}, \\
& \boldsymbol{\Theta}_{k}=\operatorname{sym}\left\{\Psi_{k} P \$_{2}^{T}\right\}-e_{6+k}^{T} S_{1} e_{6+k}-\left(e_{6+k}-e_{10+k}\right)^{T}\left(3 S_{1}\right)\left(e_{6+k}-e_{10+k}\right)-\Pi_{3-k}^{T} \frac{R_{1}}{\bar{\tau}^{2}} \Pi_{3-k}
\end{aligned}
$$

with the terms $\Pi_{3-k}, \Gamma_{3-i}(k, i=1,2)$ given in (60)-(61) and $e_{i}(1 \leq i \leq 19)$ expressed in (56). Furthermore, except for zero elements, the rest of the matrix $\boldsymbol{\Omega}=\left[\boldsymbol{\Omega}_{i j}\right]_{19 \times 19}$ can be individually listed as

$$
\begin{aligned}
& \boldsymbol{\Omega}_{\mathbf{1 1}}=Q_{1}+Q_{3}+\bar{\tau} S_{1}+\bar{h} S_{2}+\bar{\theta}^{2}(W-X)+0.25\left(\bar{\tau}^{2} R_{3}+\bar{h}^{2} R_{4}-\pi^{2} M\right)+\operatorname{sym}\left\{M_{1}^{T} A\right\}-E^{T} H \Sigma_{1} E \\
& \boldsymbol{\Omega}_{\mathbf{1 2}}=M_{1}^{T} B, \quad \boldsymbol{\Omega}_{\mathbf{1 4}}=Y F-0.25 \pi^{2} M, \quad \boldsymbol{\Omega}_{\mathbf{1 6}}=M_{1}^{T} D+E^{T} H \Sigma_{2}, \quad \boldsymbol{\Omega}_{\mathbf{1 , 1 5}}=\bar{\theta}^{2} X
\end{aligned}
$$




$$
\begin{aligned}
& \boldsymbol{\Omega}_{\mathbf{1 , 1 7}}=E^{T}(\bar{\Sigma} R-\Sigma Q) E-M_{1}^{T}+\delta A^{T} M_{1}, \boldsymbol{\Omega}_{\mathbf{1 , 1 8}}=-Y F, \quad \boldsymbol{\Omega}_{\mathbf{2 2}}=\left(\mu_{0}-1\right) Q_{1}+\left(1-\mu_{1}\right) Q_{2}-1.5 R_{3}, \\
& \boldsymbol{\Omega}_{\mathbf{2 , \mathbf { 1 7 }}}=\delta B^{T} M_{1}, \quad \boldsymbol{\Omega}_{\mathbf{3 3}}=-Q_{2}-\bar{\theta} U-9 \mathrm{~V}-1.5 R_{3}, \quad \boldsymbol{\Omega}_{\mathbf{3 5}}=3 \mathrm{~V}, \quad \boldsymbol{\Omega}_{\mathbf{3 , 1 5}}=36 \mathrm{~V}, \quad \boldsymbol{\Omega}_{\mathbf{3}, \mathbf{1 6}}=-30 \mathrm{~V}, \\
& \boldsymbol{\Omega}_{\mathbf{4 4}}=\left(2+\delta_{1} \theta_{1}+\delta_{2} \theta_{2}\right) \Phi_{2}-1.5 R_{4}-0.25 \pi^{2} M, \boldsymbol{\Omega}_{\mathbf{4}, \mathbf{1 7}}=\delta F^{T} Y^{T}, \\
& \boldsymbol{\Omega}_{\mathbf{4 , 1 8}}=-\left(1+\delta_{2} \theta_{2}\right) \Phi_{2}, \boldsymbol{\Omega}_{\mathbf{5 5}}=\bar{\theta} U-9 \mathrm{~V}-Q_{5}-1.5 R_{4}, \quad \boldsymbol{\Omega}_{\mathbf{5 , 1 5}}=-24 \mathrm{~V}, \quad \boldsymbol{\Omega}_{\mathbf{5 , 1 6}}=30 \mathrm{~V}, \\
& \boldsymbol{\Omega}_{\mathbf{6 6}}=-H, \quad \boldsymbol{\Omega}_{\mathbf{6}, \mathbf{1 7}}=\delta D^{T} M_{1}+Q-R, \quad \boldsymbol{\Omega}_{\mathbf{1 5 , 1 5}}=-192 \mathrm{~V}-\bar{\theta}^{2}(4 W+X), \\
& \boldsymbol{\Omega}_{\mathbf{1 5 , 1 6}}=180 \mathrm{~V}+3 \bar{\theta}^{2} W, \quad \boldsymbol{\Omega}_{\mathbf{1 6}, \mathbf{1 6}}=-180 \mathrm{~V}-3 \bar{\theta}^{2} W \\
& \boldsymbol{\Omega}_{\mathbf{1 7}, \mathbf{1 7}}=\bar{\tau} R_{1}+\bar{h} R_{2}+\bar{\theta}^{2} V+0.25 \bar{\delta}^{2} X+N_{6}+\bar{h}^{2} M-\operatorname{sym}\left\{\delta M_{1}^{T}\right\}+Q_{4}, \\
& \boldsymbol{\Omega}_{\mathbf{1 7}, \mathbf{1 8}}=-\delta Y F, \quad \boldsymbol{\Omega}_{\mathbf{1 8 , 1 8}}=-\left(\delta_{1}+\delta_{2}\right) \Phi_{1}+\left(1+\delta_{2} \theta_{2}\right) \Phi_{2}, \\
& \boldsymbol{\Omega}_{\mathbf{1 9 , 1 9}}=-Q_{6}-N_{6}, \quad \boldsymbol{\Omega}_{\mathbf{7 7}}=\boldsymbol{\Omega}_{\mathbf{8 8}}=-3 R_{3}, \quad \boldsymbol{\Omega}_{\mathbf{2}, \mathbf{1 1}}=\boldsymbol{\Omega}_{\mathbf{3}, \mathbf{1 2}}=1.5 R_{3} \text {, } \\
& \boldsymbol{\Omega}_{\mathbf{7 , 1 1}}=\boldsymbol{\Omega}_{\mathbf{8 , 1 2}}=3 R_{3}, \quad \boldsymbol{\Omega}_{\mathbf{1 1 , 1 1}}=\boldsymbol{\Omega}_{\mathbf{1 2}, \mathbf{1 2}}=-4.5 R_{3}, \quad \boldsymbol{\Omega}_{\mathbf{9 9}}=\boldsymbol{\Omega}_{\mathbf{1 0 , 1 0}}=-3 R_{4}, \\
& \boldsymbol{\Omega}_{\mathbf{4}, \mathbf{1 3}}=\boldsymbol{\Omega}_{\mathbf{5 , 1 4}}=1.5 R_{4}, \quad \boldsymbol{\Omega}_{\mathbf{9 , 1 3}}=\boldsymbol{\Omega}_{\mathbf{1 0 , 1 4}}=3 R_{4}, \quad \boldsymbol{\Omega}_{\mathbf{1 3 , 1 3}}=\boldsymbol{\Omega}_{\mathbf{1 4 , 1 4}}=-4.5 R_{4} \text {. }
\end{aligned}
$$

Then the master system (1) and the slave one (2) can achieve the desired synchronization. Moreover, the corresponding controller gain in (6) can be computed out as $K=M_{1}^{-T} Y$.

Proof. Now based on the assumptions H1-H2 and (7), we can construct the Lyapunov-Krasovskii functional as

$$
V\left(\varepsilon_{t}\right)=\sum_{i=1}^{6} V_{i}\left(\varepsilon_{t}\right), \quad \forall t \in \mathbf{\Xi}_{l},
$$

with

$$
\begin{aligned}
V_{1}\left(\varepsilon_{t}\right)= & \zeta^{T}(t) P \zeta(t)+\frac{\alpha^{2}(t)}{2}+\frac{\beta^{2}(t)}{2}+2 \sum_{i=1}^{n}\left\{q_{i} \int_{0}^{E_{i} \varepsilon(t)}\left[f_{i}(s)-\sigma_{i}^{-} s\right] d s+r_{i} \int_{0}^{E_{i} \varepsilon(t)}\left[\sigma_{i}^{+} s-f_{i}(s)\right] d s\right\}, \\
V_{2}\left(\varepsilon_{t}\right)= & \int_{t-\tau(t)}^{t} \varepsilon^{T}(s) Q_{1} \varepsilon(s) d s+\int_{t-\bar{\tau}}^{t-\tau(t)} \varepsilon^{T}(s) Q_{2} \varepsilon(s) d s+\int_{t-\eta(t)}^{t}\left[\varepsilon^{T}(s) Q_{3} \varepsilon(s)+\dot{\varepsilon}^{T}(s) Q_{4} \dot{\varepsilon}(s)\right] d s \\
& +\int_{t-\bar{h}}^{t-\eta(t)}\left[\varepsilon^{T}(s) Q_{5} \varepsilon(s)+\dot{\varepsilon}^{T}(s) Q_{6} \dot{\varepsilon}(s)\right] d s, \\
V_{3}\left(\varepsilon_{t}\right)= & \int_{-\bar{\tau}}^{0} \int_{t+s}^{t}\left[\dot{\varepsilon}^{T}(\theta) R_{1} \dot{\varepsilon}(\theta)+\varepsilon^{T}(\theta) S_{1} \varepsilon(\theta)\right] d \theta d s+\int_{-\bar{h}}^{0} \int_{t+s}^{t}\left[\dot{\varepsilon}^{T}(\theta) R_{2} \dot{\varepsilon}(\theta)+\varepsilon^{T}(\theta) S_{2} \varepsilon(\theta)\right] d \theta d s \\
& +\frac{1}{2} \int_{t-\bar{\tau}}^{t} \int_{t-\bar{\tau}}^{\varrho} \int_{\theta}^{t} \dot{\varepsilon}^{T}(s) R_{3} \dot{\varepsilon}(s) d s d \theta d \varrho+\frac{1}{2} \int_{t-\bar{h}}^{t} \int_{t-\bar{h}}^{\int_{\theta}} \int_{\theta}^{\dot{\varepsilon}^{T}(s) R_{4} \dot{\varepsilon}(s) d s d \theta d \varrho,} \\
V_{4}\left(\varepsilon_{t}\right)= & \bar{\theta} \int_{t-\bar{\tau}}^{t-\bar{h}} \varepsilon^{T}(s) U \varepsilon(s) d s+\bar{\theta} \int_{-\bar{\tau}}^{-\bar{h}} \int_{t+s}^{t}\left[\dot{\varepsilon}^{T}(\theta) V \dot{\varepsilon}(\theta)+\varepsilon^{T}(\theta) W \varepsilon(\theta)\right] d \theta d s+\frac{\bar{\delta}}{2} \int_{t-\bar{\tau}}^{t-\bar{h}} \int_{\varrho}^{0} \int_{t+\theta}^{t} \dot{\varepsilon}^{T}(s) X \dot{\varepsilon}(s) d s d \theta d \varrho, \\
V_{5}\left(\varepsilon_{t}\right)= & \int_{t-\bar{h}}^{t} \dot{\varepsilon}(s) N_{6} \dot{\varepsilon}(s) d s+\zeta^{T}(t)\left[\bar{h}\left(N_{1}+\frac{\bar{h}^{2}}{3} N_{4}-\operatorname{sym}\left\{\left[N_{5} N_{5} 0\right]\right\}\right)+\operatorname{sym}\left\{\left[N_{3}-N_{3} 2 N_{5}\right]\right\}\right] \zeta(t), \\
V_{6}\left(\varepsilon_{t}\right)= & \bar{h}^{2} \int_{i_{l}}^{t} \dot{\varepsilon}(s) M \dot{\varepsilon}(s) d s-\frac{\pi^{2}}{4} \int_{i_{l}}^{t}\left[\varepsilon(s)-\varepsilon\left(i_{l}\right)\right]^{T} M\left[\varepsilon(s)-\varepsilon\left(i_{l}\right)\right] d s,
\end{aligned}
$$


where $V_{i}\left(\varepsilon_{t}\right)(i=5,6)$ are discontinuous functionals and

$$
\zeta^{T}(t)=\left[\varepsilon^{T}(t), \varepsilon^{T}(t-\bar{h}), \int_{t-\bar{h}}^{t} \varepsilon^{T}(s) d s \int_{t-\bar{\tau}}^{t} \varepsilon^{T}(s) d s\right] .
$$

Especially, the positive definiteness and superiorities of $V_{i}\left(\varepsilon_{t}\right)(i=5,6)$ can be checked in the Refs. $[13,15,16]$.

Then together with the denotations in (51)-(59) and $\boldsymbol{\Xi}$ in (12), the derivative of $V_{i}\left(\varepsilon_{t}\right)(i=1,2, \cdots, 6)$ along the system (11) can be directly computed out as

$$
\begin{aligned}
& \dot{V}_{1}\left(\varepsilon_{t}\right)= 2 \zeta^{T}(t) P \dot{\zeta}(t)+\alpha(t) \dot{\alpha}(t)+\beta(t) \dot{\beta}(t)+2 f^{T}(E \varepsilon(t))(Q-R) E \dot{\varepsilon}(t)-2 \varepsilon^{T}(t) E^{T}(\Sigma Q-\bar{\Sigma} R) E \dot{\varepsilon}(t), \\
& \dot{V}_{2}\left(\varepsilon_{t}\right)= \varepsilon^{T}(t)\left(Q_{1}+Q_{3}\right) \varepsilon(t)-(1-\dot{\tau}(t)) \varepsilon^{T}(t-\tau(t))\left(Q_{1}-Q_{2}\right) \varepsilon(t-\tau(t))-\varepsilon^{T}(t-\bar{\tau}) Q_{2} \varepsilon(t-\bar{\tau}) \\
&+\dot{\varepsilon}^{T}(t) Q_{4} \dot{\varepsilon}(t)-\varepsilon^{T}(t-\bar{h}) Q_{5} \varepsilon(t-\bar{h})-\dot{\varepsilon}^{T}(t-\bar{h}) Q_{6} \dot{\varepsilon}(t-\bar{h}), \\
& \dot{V}_{3}\left(\varepsilon_{t}\right)= \varepsilon^{T}(t)\left(\bar{\tau} S_{1}+\bar{h} S_{2}+0.25 \bar{\tau}^{2} R_{3}+0.25 \bar{h}^{2} R_{4}\right) \varepsilon(t)-\int_{t-\bar{\tau}}^{t}\left[\dot{\varepsilon}^{T}(\theta) R_{1} \dot{\varepsilon}(\theta)+\varepsilon^{T}(\theta) S_{1} \varepsilon(\theta)\right] d \theta \\
&+\dot{\varepsilon}^{T}(t)\left(\bar{\tau} R_{1}+\bar{h} R_{2}\right) \dot{\varepsilon}(t)-\int_{t-\bar{h}}^{t}\left[\dot{\varepsilon}^{T}(\theta) R_{2} \dot{\varepsilon}(\theta)+\varepsilon^{T}(\theta) S_{2} \varepsilon(\theta)\right] d \theta-\frac{1}{2} \int_{t-\bar{\tau}}^{t} \int_{t-\bar{\tau}}^{\theta} \dot{\varepsilon}^{T}(s) R_{3} \dot{\varepsilon}(s) d s d \theta \\
&-\frac{1}{2} \int_{t-\bar{h}}^{t} \int_{t-\bar{h}}^{\theta} \dot{\varepsilon}^{T}(s) R_{4} \dot{\varepsilon}(s) d s d \theta, \\
& \dot{V}_{4}\left(\varepsilon_{t}\right)= \varepsilon^{T}(t-\bar{h})(\bar{\theta} U) \varepsilon(t-\bar{h})-\varepsilon^{T}(t-\bar{\tau})(\bar{\theta} U) \varepsilon(t-\bar{\tau})+\dot{\varepsilon}^{T}(t)\left(\bar{\theta}^{2} V+0.25 \bar{\delta}^{2} X\right) \dot{\varepsilon}(t)+\varepsilon^{T}(t)\left(\bar{\theta}^{2} W\right) \varepsilon(t) \\
&-\bar{\theta} \int_{t-\bar{h}}^{t-\bar{\tau}}\left[\dot{\varepsilon}^{T}(\theta) V \dot{\varepsilon}(\theta)+\varepsilon^{T}(\theta) W \varepsilon(\theta)\right] d \theta-\frac{\bar{\delta}}{2} \int_{t-\bar{\tau}}^{t-\bar{h}} \int_{t+\theta}^{t} \dot{\varepsilon}^{T}(s) X \dot{\varepsilon}(s) d s d \varrho, \\
& \dot{V}_{5}\left(\varepsilon_{t}\right)= \\
& \dot{\varepsilon}^{T}(t) N_{6} \dot{\varepsilon}(t)-\dot{\varepsilon}^{T}(t-\bar{h}) N_{6} \dot{\varepsilon}(t-\bar{h})+\xi^{T}(t) \operatorname{sym}\left\{\Upsilon_{0} \Xi \$_{1}^{T}+\eta(t) \Upsilon_{1} \boldsymbol{\Xi} \$_{1}^{T}+\bar{\eta}(t) \Upsilon_{2} \Xi \$_{1}^{T}\right\} \xi(t), \\
& \dot{V}_{6}\left(\varepsilon_{t}\right)= \bar{h}^{2} \dot{\varepsilon}^{T}(t) M \dot{\varepsilon}(t)-\frac{\pi^{2}}{4}\left[\varepsilon(t)-\varepsilon\left(i_{l}\right)\right]^{T} M\left[\varepsilon(t)-\varepsilon\left(i_{l}\right)\right] .
\end{aligned}
$$

Furthermore, as for some terms in (14) and (16)-(17), we can respectively utilize the conditions in (8)-(9), Lemmas $1-4$, and denotations in (51)-(61) to give their estimations as

$$
\begin{aligned}
2 \zeta^{T}(t) P \dot{\zeta}(t)= & \xi^{T}(t) \mathbf{s y m}\left\{\Lambda P \$_{2}^{T}+\tau(t) \Psi_{1} P \$_{2}^{T}+\bar{\tau}(t) \Psi_{2} P \$_{2}^{T}+\eta(t) \Phi_{1} P \$_{2}^{T}+\bar{\eta}(t) \Phi_{2} P \$_{2}^{T}\right\} \xi(t), \\
\alpha(t) \dot{\alpha}(t)+\beta(t) \dot{\beta}(t) \leq & \varepsilon^{T}\left(t_{l}\right) \Phi_{2} \varepsilon\left(t_{l}\right)+\frac{\beta(t)-\theta_{1}}{\alpha(t)} \varepsilon^{T}\left(i_{l}\right) \Phi_{2} \varepsilon\left(i_{l}\right)-\delta_{1}\left[e^{T}\left(i_{l}\right) \Phi_{1} e\left(i_{l}\right)-\theta_{1} \varepsilon^{T}\left(i_{l}\right) \Phi_{2} \varepsilon\left(i_{l}\right)\right] \\
& +\varepsilon^{T}\left(i_{l}\right) \Phi_{2} \varepsilon\left(i_{l}\right)+\frac{\alpha(t)-\theta_{2}}{\beta(t)} \varepsilon^{T}\left(t_{l}\right) \Phi_{2} \varepsilon\left(t_{l}\right)-\delta_{2}\left[e^{T}\left(i_{l}\right) \Phi_{1} e\left(i_{l}\right)-\theta_{2} \varepsilon^{T}\left(t_{l}\right) \Phi_{2} \varepsilon\left(t_{l}\right)\right] \\
\leq & \varepsilon^{T}\left(t_{l}\right)\left(1+\delta_{2} \theta_{2}\right) \Phi_{2} \varepsilon\left(t_{l}\right)+\varepsilon^{T}\left(i_{l}\right)\left(1+\delta_{1} \theta_{1}\right) \Phi_{2} \varepsilon\left(i_{l}\right)-e^{T}\left(i_{l}\right)\left(\delta_{1}+\delta_{2}\right) \Phi_{1} e\left(i_{l}\right) \\
= & -e^{T}\left(i_{l}\right)\left(\delta_{1}+\delta_{2}\right) \Phi_{1} e\left(i_{l}\right)+\left[\varepsilon(t-\eta(t))-e\left(i_{l}\right)\right]^{T}\left(1+\delta_{2} \theta_{2}\right) \Phi_{2}\left[\varepsilon(t-\eta(t))-e\left(i_{l}\right)\right] \\
& +\varepsilon^{T}(t-\eta(t))\left(1+\delta_{1} \theta_{1}\right) \Phi_{2} \varepsilon(t-\eta(t)), \\
-\int_{t-\bar{\tau}}^{t} \dot{\varepsilon}^{T}(\theta) R_{1} \dot{\varepsilon}(\theta) d \theta \leq & -\frac{1}{\bar{\tau}} \xi^{T}(t)\left[\begin{array}{l}
\Pi_{1} \\
\Pi_{2}
\end{array}\right]^{T}\left(\left[\begin{array}{cc}
\bar{R}_{1} & U_{1} \\
* & \bar{R}_{1}
\end{array}\right]+\left[\begin{array}{cc}
\frac{\bar{\tau}(t)}{\bar{\tau}} & 0 \\
0 & \frac{\tau(t)}{\bar{\tau}}
\end{array}\right] \otimes\left(\bar{R}_{1}-U_{1} \bar{R}_{1}^{-1} U_{1}^{T}\right)\right)\left[\begin{array}{l}
\Pi_{1} \\
\Pi_{2}
\end{array}\right] \xi(t), \\
-\int_{t-\bar{\tau}}^{t} \varepsilon^{T}(\theta) S_{1} \varepsilon(\theta) d \theta \leq & -\tau(t) \rho^{T}(t) S_{1} \rho(t)-\tau(t)[\rho(t)-\nu(t)]^{T}\left(3 S_{1}\right)[\rho(t)-\nu(t)]-\bar{\tau}(t) \sigma^{T}(t) S_{1} \sigma(t)
\end{aligned}
$$




$$
\begin{aligned}
& -\bar{\tau}(t)[\sigma(t)-\omega(t)]^{T}\left(3 S_{1}\right)[\sigma(t)-\omega(t)], \\
-\int_{t-\bar{h}}^{t} \dot{\varepsilon}^{T}(\theta) R_{2} \dot{\varepsilon}(\theta) d \theta \leq & -\frac{1}{\bar{h}} \xi^{T}(t)\left[\begin{array}{l}
\Gamma_{1} \\
\Gamma_{2}
\end{array}\right]^{T}\left(\left[\begin{array}{cc}
\bar{R}_{2} & U_{2} \\
* & \bar{R}_{2}
\end{array}\right]+\left[\begin{array}{cc}
\frac{\bar{\eta}(t)}{h} & 0 \\
0 & \frac{\eta(t)}{h}
\end{array}\right] \otimes\left(\bar{R}_{2}-U_{2} \bar{R}_{2}^{-1} U_{2}^{T}\right)\right)\left[\begin{array}{l}
\Gamma_{1} \\
\Gamma_{2}
\end{array}\right] \xi(t), \\
-\int_{t-\bar{h}}^{t} \varepsilon^{T}(\theta) S_{2} \varepsilon(\theta) d \theta \leq & -\eta(t) \varrho^{T}(t) S_{2} \varrho(t)-\eta(t)[\varrho(t)-\phi(t)]^{T}\left(3 S_{2}\right)[\varrho(t)-\phi(t)]-\bar{\eta}(t) \theta^{T}(t) S_{2} \theta(t) \\
& -\bar{\eta}(t)[\theta(t)-\varphi(t)]^{T}\left(3 S_{2}\right)[\theta(t)-\varphi(t)], \\
-\bar{\theta} \int_{t-\bar{\tau}}^{t-\bar{h}} \dot{\varepsilon}^{T}(\theta) V \dot{\varepsilon}(\theta) d \theta \leq & -[\varepsilon(t-\bar{h})-\varepsilon(t-\bar{\tau})]^{T} V[\varepsilon(t-\bar{h})-\varepsilon(t-\bar{\tau})] \\
& -[\varepsilon(t-\bar{h})+\varepsilon(t-\bar{\tau})-2 \psi(t)]^{T}(3 V)[\varepsilon(t-\bar{h})+\varepsilon(t-\bar{\tau})-2 \psi(t)] \\
& -[\varepsilon(t-h)-\varepsilon(t-\bar{\tau})+6 \psi(t)-6 \chi(t)]^{T}(5 V)[\varepsilon(t-h)-\varepsilon(t-\bar{\tau})+6 \psi(t)-6 \chi(t)], \\
-\bar{\theta} \int_{t-\bar{\tau}}^{t-h} \varepsilon^{T}(\theta) W \varepsilon(\theta) d \theta \leq & -\bar{\theta}^{2} \psi^{T}(t) W \psi(t)-\bar{\theta}^{2}[\psi(t)-\chi(t)]^{T}(3 W)[\psi(t)-\chi(t)] .
\end{aligned}
$$

Especially, as for the double integral terms in (16)-(17), it follows from Lemma 2 and Lemma 3 that

$$
\begin{aligned}
-\frac{1}{2} \int_{t-\bar{\tau}}^{t} \int_{t-\bar{\tau}}^{\theta} \dot{\varepsilon}^{T}(s) R_{3} \dot{\varepsilon}(s) d s d \theta= & -\frac{1}{2}\left[\int_{t-\tau(t)}^{t} \int_{t-\bar{\tau}}^{t-\tau(t)}+\int_{t-\tau(t)}^{t} \int_{t-\tau(t)}^{\theta}+\int_{t-\bar{\tau}}^{t-\tau(t)} \int_{t-\bar{\tau}}^{\theta}\right] \dot{\varepsilon}^{T}(s) R_{3} \dot{\varepsilon}(s) d s d \theta \\
\leq & -[\rho(t)-\varepsilon(t-\tau(t))]^{T} R_{3}[\rho(t)-\varepsilon(t-\tau(t))] \\
& -[\sigma(t)-\varepsilon(t-\bar{\tau})]^{T} R_{3}[\sigma(t)-\varepsilon(t-\bar{\tau})] \\
& -\left[\frac{1}{2} \varepsilon(t-\tau(t))+\rho(t)-\frac{3}{2} \nu(t)\right]\left(2 R_{3}\right)\left[\frac{1}{2} \varepsilon(t-\tau(t))+\rho(t)-\frac{3}{2} \nu(t)\right] \\
& -\left[\frac{1}{2} \varepsilon(t-\bar{\tau})+\sigma(t)-\frac{3}{2} \omega(t)\right]^{T}\left(2 R_{3}\right)\left[\frac{1}{2} \varepsilon(t-\bar{\tau})+\sigma(t)-\frac{3}{2} \omega(t)\right], \\
-\frac{1}{2} \int_{t-\bar{h}}^{t} \int_{t-\bar{h}}^{\theta} \dot{\varepsilon}^{T}(s) R_{4} \dot{\varepsilon}(s) d s d \theta= & -\frac{1}{2}\left[\int_{t-\eta(t)}^{t} \int_{t-\bar{h}}^{t-\eta(t)}+\int_{t-\eta(t)}^{t}+\int_{t-\eta(t)}^{\theta} \int_{t-\bar{h}}^{\theta}\right] \dot{\varepsilon}^{T}(s) R_{4} \dot{\varepsilon}(s) d s d \theta \\
\leq & -[\varrho(t)-\varepsilon(t-\eta(t))]^{T} R_{4}[\varrho(t)-\varepsilon(t-\eta(t))] \\
& -[\vartheta(t)-\varepsilon(t-\bar{h})]^{T} R_{4}[\vartheta(t)-\varepsilon(t-\bar{h})] \\
& -\left[\frac{1}{2} \varepsilon(t-\eta(t))+\varrho(t)-\frac{3}{2} \phi(t)\right]\left(2 R_{4}\right)\left[\frac{1}{2} \varepsilon(t-\eta(t))+\varrho(t)-\frac{3}{2} \phi(t)\right] \\
& -\left[\frac{1}{2} \varepsilon(t-\bar{h})+\vartheta(t)-\frac{3}{2} \varphi(t)\right]^{T}\left(2 R_{4}\right)\left[\frac{1}{2} \varepsilon(t-\bar{h})+\vartheta(t)-\frac{3}{2} \varphi(t)\right], \\
\bar{\delta} \int_{t-\bar{\tau}}^{t-\bar{h}} \int_{t+\theta}^{t} \dot{\varepsilon}^{T}(s) X \dot{\varepsilon}(s) d s d \varrho \leq & -\bar{\theta}^{2}[\varepsilon(t)-\psi(t)]^{T} X[\varepsilon(t)-\psi(t)] .
\end{aligned}
$$

In what is next, for any $n \times n$ matrix $M_{1}$, diagonal one $H>0$, and a scalar $\delta>0$, it follows from (11) and $\mathbf{H 2}$ that

$$
0=2\left[\varepsilon^{T}(t) M_{1}^{T}+\dot{\varepsilon}^{T}(t)\left(\delta M_{1}^{T}\right)\right]\left[-\dot{\varepsilon}(t)+A \varepsilon(t)+B \varepsilon(t-\tau(t))+D f(E \varepsilon(t))+K F \varepsilon(t-\eta(t))-K F e\left(i_{l}\right)\right],
$$




$$
0 \leq-\left[\varepsilon^{T}(t) E^{T} H \Sigma_{1} E \varepsilon(t)-2 \varepsilon^{T}(t) E^{T} H \Sigma_{2} f(E \varepsilon(t))+f^{T}(E \varepsilon(t)) H f(E \varepsilon(t))\right] .
$$

Now combining with the right terms from (14) to (32), we can verify that $\dot{V}\left(\varepsilon_{t}\right)$ satisfies

$$
\begin{aligned}
\dot{V}\left(\varepsilon_{t}\right) \leq & \xi^{T}(t)\left\{\boldsymbol{\Omega}+\boldsymbol{\Upsilon}+\eta(t) \mathbf{\Psi}_{1}+\bar{\eta}(t) \mathbf{\Psi}_{2}+\tau(t) \boldsymbol{\Theta}_{1}+\bar{\tau}(t) \boldsymbol{\Theta}_{2}+\frac{\eta(t)}{\bar{h}^{2}} \Gamma_{2}^{T} U_{2} \bar{R}_{2}^{-1} U_{2}^{T} \Gamma_{2}+\frac{\bar{\eta}(t)}{\bar{h}^{2}} \Gamma_{1}^{T} U_{2} \bar{R}_{2}^{-1} U_{2}^{T} \Gamma_{1}\right. \\
& \left.+\frac{\tau(t)}{\bar{\tau}^{2}} \Pi_{2}^{T} U_{1} \bar{R}_{1}^{-1} U_{1}^{T} \Pi_{2}+\frac{\bar{\tau}(t)}{\bar{\tau}^{2}} \Pi_{1}^{T} U_{1} \bar{R}_{1}^{-1} U_{1}^{T} \Pi_{1}+\left[\dot{\tau}(t)-\mu_{0}\right] \boldsymbol{\Phi}_{1}+\left[\mu_{1}-\dot{\tau}(t)\right] \boldsymbol{\Phi}_{2}\right\} \xi(t) \\
\doteq & \xi^{T}(t) \boldsymbol{\Delta}(t) \xi(t),
\end{aligned}
$$

where the terms $\boldsymbol{\Omega}, \boldsymbol{\Upsilon}, \mathbf{\Psi}_{1}, \mathbf{\Psi}_{2}, \boldsymbol{\Theta}_{1}, \boldsymbol{\Theta}_{2}, \boldsymbol{\Phi}_{1}, \boldsymbol{\Phi}_{2}$ are respectively presented in (12) and the ones $\bar{\tau}(t), \bar{\eta}(t)$ are defined in (51). Together with the definition on Schur-complement, as for $i, j, k \in\{1,2\}$, we can check that the LMI in (12) can guarantee the following term to be true

$$
\boldsymbol{\Omega}+\boldsymbol{\Upsilon}+\bar{\mu} \boldsymbol{\Phi}_{i}+\bar{h}\left[\boldsymbol{\Psi}_{j}+\frac{1}{\bar{h}^{2}} \Gamma_{3-j}^{T} U_{2} \bar{R}_{2}^{-1} U_{2}^{T} \Gamma_{3-j}\right]+\bar{\tau}\left[\boldsymbol{\Theta}_{k}+\frac{1}{\bar{\tau}^{2}} \Pi_{3-k}^{T} U_{1} \bar{R}_{1}^{-1} U_{1}^{T} \Pi_{3-k}\right]<0 .
$$

Now based on Lemma 5 , the terms in (34) can be equivalent to $\boldsymbol{\Delta}(t)<0$. Therefore, once the condition (12) holds, there must exist a positive scalar $\epsilon>$ such that

$$
\dot{V}\left(\varepsilon_{t}\right) \leq-\xi^{T}(t) \boldsymbol{\Delta}(t) \xi(t) \leq-\epsilon\|\varepsilon(t)\|^{2}<0, \forall \varepsilon(t) \neq 0 .
$$

Then the error system (11) is absolutely stable, i.e., the systems (1)-(2) achieve the desired synchronization. It completes the proof.

Remark 3. Once $\alpha(t), \beta(t)$ are given constants, the event-triggered scheme (7) can be reduced to a traditional one

$$
e^{T}\left(i_{s}\right) \Phi_{1} e\left(i_{s}\right) \leq \alpha \varepsilon^{T}\left(t_{l}\right) \Phi_{2} \varepsilon\left(t_{l}\right)+\beta \varepsilon^{T}\left(i_{s}\right) \Phi_{2} \varepsilon\left(i_{s}\right) .
$$

Then based on the scheme in (35) and Theorem 1, we also can derive the relevant results.

Most recently, a kind of novel delay-product-type LKF was put forward [18,46], which can help reduce the conservatism more effectively than ever. The novelties of this technique benefits from that the advantages of Wirtingerbased integral inequalities can be fully utilized by resorting to introducing some necessary integral vectors in the LKF derivative. Thus in what follows, we also use this kind of LKF to derive the relevant results. Prior to presenting the theorem, we firstly introduce some denotations as

$$
\begin{aligned}
& \varsigma^{T}(t)=\left[\begin{array}{lllllllllllll}
\varepsilon^{T}(t) & \varepsilon^{T}(t-\tau(t)) & \varepsilon^{T}(t-\bar{\tau}) & \varepsilon^{T}\left(i_{l}\right) & \varepsilon^{T}(t-\bar{h}) & f^{T}(E \varepsilon(t)) & \rho^{T}(t) & \sigma^{T}(t) & \varrho^{T}(t) & \theta^{T}(t) & \nu^{T}(t) & \omega^{T}(t)
\end{array}\right.
\end{aligned}
$$

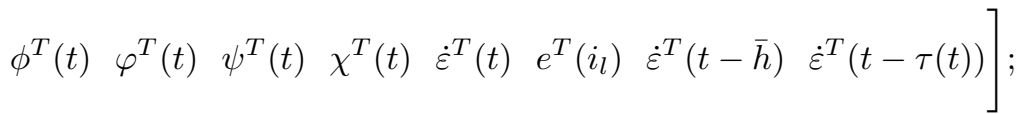

$$
\begin{aligned}
& e_{i}=\left[\begin{array}{lll}
0_{n \times(i-1) n} & I_{n} & 0_{n \times(20-i) n}
\end{array}\right]^{T} \quad(1 \leq i \leq 20) ; \\
& \bar{\Psi}_{1}(t)=\left[e_{1}, e_{2}, \tau(t) e_{7}, \bar{\tau}(t) e_{8}\right], \quad \bar{\Psi}_{2}(t)=\left[e_{17},(1-\dot{\tau}(t)) e_{20}, e_{1}-(1-\dot{\tau}(t)) e_{2},(1-\dot{\tau}(t)) e_{2}-e_{3}\right] ; \\
& \hat{\Psi}_{1}=\left[e_{1}, e_{2}, e_{7}\right], \quad \hat{\Psi}_{2}(t)=\left[\tau(t) e_{17}, \tau(t)(1-\dot{\tau}(t)) e_{20}, e_{1}-(1-\dot{\tau}(t)) e_{2}-\dot{\tau}(t) e_{7}\right], \check{\Psi}_{1}=\left[e_{1}, e_{17}\right] \text {; } \\
& \tilde{\Psi}_{1}=\left[e_{1}, e_{2}, e_{8}\right], \quad \tilde{\Psi}_{2}(t)=\left[\bar{\tau}(t) e_{17}, \bar{\tau}(t)(1-\dot{\tau}(t)) e_{20},(1-\dot{\tau}(t)) e_{2}-e_{3}+\dot{\tau}(t) e_{8}\right], \check{\Psi}_{2}=\left[e_{2}, e_{20}\right] .
\end{aligned}
$$

Theorem 2. For given scalars $\bar{\tau} \geq 0, \bar{h} \geq 0, \bar{\theta} \geq 0, \bar{\delta} \geq 0, \delta \geq 0, \delta_{i}>0, \theta_{i}>0(i=1,2), \mu_{0}, \mu_{1}$ in (2) and (51), if there exist matrices $P>0, P_{h}>0(h=1,2), Q_{i}>0(i=1,2, \cdots, 6), R_{j}>0(j=1,2,3,4), S_{l}>0, \Phi_{l}>$ $0, U_{l}(l=1,2), U>0, V>0, W>0, M>0, X>0, M_{1}, N_{m}(m=1,2, \cdots, 6)$ making $\bar{R}_{l}=\operatorname{diag}\left(R_{l}, 3 R_{l}, 5 R_{l}\right)$, 
$\left[\begin{array}{cc}\bar{R}_{l} & U_{l} \\ * & \bar{R}_{l}\end{array}\right] \geq 0(l=1,2),\left[\begin{array}{ccc}N_{1} & N_{2} & N_{3} \\ * & N_{4} & N_{5} \\ * & * & N_{6}\end{array}\right] \geq 0$, and diagonal matrices $Q>0, R>0, H>0$ such that the LMIs in (41)-(42) hold

$$
\begin{gathered}
{\left[\begin{array}{ccc}
\left.\Phi(0,0, \dot{\tau}(t))\right|_{\dot{\tau}(t)=\mu_{0}, \mu_{m}} & \Pi_{1}^{T} U_{1} & \Gamma_{1}^{T} U_{2} \\
* & -\bar{\tau} \bar{R}_{1} & 0 \\
* & * & -\bar{h} \bar{R}_{2}
\end{array}\right]<0,\left[\begin{array}{ccc}
\left.\boldsymbol{\Phi}(\bar{\tau}, 0, \dot{\tau}(t))\right|_{\dot{\tau}(t)=\mu_{0}, \mu_{m}} & \Pi_{2}^{T} U_{1} & \Gamma_{1}^{T} U_{2} \\
* & -\bar{\tau} \bar{R}_{1} & 0 \\
* & * & -\bar{h} \bar{R}_{2}
\end{array}\right]<0,} \\
{\left[\begin{array}{ccc}
\left.\Phi(0, \bar{h}, \dot{\tau}(t))\right|_{\dot{\tau}(t)=\mu_{0}, \mu_{m}} & \Pi_{1}^{T} U_{1} & \Gamma_{2}^{T} U_{2} \\
* & -\bar{\tau} \bar{R}_{1} & 0 \\
* & * & -\bar{h} \bar{R}_{2}
\end{array}\right]<0,}
\end{gathered}
$$

where $\boldsymbol{\Phi}(\tau(t), \eta(t), \dot{\tau}(t))=\mathbf{\Omega}+\mathbf{\Upsilon}_{1}+\mathbf{\Upsilon}_{2}(t)+\eta(t) \mathbf{\Psi}_{1}+\bar{\eta}(t) \mathbf{\Psi}_{2}+\tau(t) \boldsymbol{\Theta}_{1}+\bar{\tau}(t) \boldsymbol{\Theta}_{2}$ and

$$
\begin{aligned}
& \boldsymbol{\Upsilon}_{1}= \check{\Psi}_{1} Q_{1} \check{\Psi}_{1}^{T}-\frac{1}{\bar{\tau}}\left[\begin{array}{l}
\Pi_{1} \\
\Pi_{2}
\end{array}\right]^{T}\left[\begin{array}{cc}
\bar{R}_{1} & U_{1} \\
* & \bar{R}_{1}
\end{array}\right]\left[\begin{array}{l}
\Pi_{1} \\
\Pi_{2}
\end{array}\right]-\frac{1}{\bar{h}}\left[\begin{array}{c}
\Gamma_{1} \\
\Gamma_{2}
\end{array}\right]^{T}\left[\begin{array}{cc}
\bar{R}_{2} & U_{2} \\
* & \bar{R}_{2}
\end{array}\right]\left[\begin{array}{c}
\Gamma_{1} \\
\Gamma_{2}
\end{array}\right], \\
& \boldsymbol{\Upsilon}_{2}(t)=\operatorname{sym}\left(\bar{\Psi}_{1}(t) P \bar{\Psi}_{2}^{T}(t)+\hat{\Psi}_{1} P_{1} \hat{\Psi}_{2}^{T}(t)+\tilde{\Psi}_{1} P_{2} \tilde{\Psi}_{2}^{T}(t)+\Upsilon_{0} \boldsymbol{\Xi} \$_{1}^{T}\right)+\hat{\Psi}_{1}\left(\dot{\tau}(t) P_{1}\right) \hat{\Psi}_{1}^{T}-\tilde{\Psi}_{1}\left(\dot{\tau}(t) P_{2}\right) \tilde{\Psi}_{1}^{T} \\
& \\
& \quad-(1-\dot{\tau}(t)) \check{\Psi}_{1}\left(Q_{1}-\left[\begin{array}{cc}
Q_{2} & 0 \\
* & 0
\end{array}\right]\right) \check{\Psi}_{1}^{T}, \\
& \boldsymbol{\Psi}_{j}=\operatorname{sym}\left\{\Upsilon_{j} \boldsymbol{\Xi}_{1}^{T}\right\}-e_{8+j}^{T} S_{2} e_{8+j}-\left(e_{8+j}-e_{12+j}\right)^{T}\left(3 S_{2}\right)\left(e_{8+j}-e_{12+j}\right)-\Gamma_{3-j}^{T} \frac{R_{2}}{\bar{h}^{2}} \Gamma_{3-j}, \\
& \boldsymbol{\Theta}_{k}=-e_{6+k}^{T} S_{1} e_{6+k}-\left(e_{6+k}-e_{10+k}\right)^{T}\left(3 S_{1}\right)\left(e_{6+k}-e_{10+k}\right)-\Pi_{3-k}^{T} \frac{R_{1}}{\bar{\tau}^{2}} \Pi_{3-k}
\end{aligned}
$$

with $\bar{\Psi}_{1}(t), \bar{\Psi}_{2}(t), \hat{\Psi}_{1}, \hat{\Psi}_{2}(t), \tilde{\Psi}_{1}, \tilde{\Psi}_{2}(t), \check{\Psi}_{1}, \check{\Psi}_{2}$ denoted in (38)-(40), the terms $\Pi_{3-k}, \Gamma_{3-i}(k, i=1,2)$ given in (60)(61), and $\boldsymbol{\Xi}$ is presented in (12). Furthermore, except for zero terms and the identical ones in Theorem 1, the rest of the matrix $\boldsymbol{\Omega}=\left[\boldsymbol{\Omega}_{i j}\right]_{20 \times 20}$ can be presented as

$$
\boldsymbol{\Omega}_{\mathbf{1 1}}=Q_{3}+\bar{\tau} S_{1}+\bar{h} S_{2}+\bar{\theta}^{2}(W-X)+0.25\left(\bar{\tau}^{2} R_{3}+\bar{h}^{2} R_{4}-\pi^{2} M\right)+\operatorname{sym}\left\{M_{1}^{T} A\right\}-E^{T} H \Sigma_{1} E, \boldsymbol{\Omega}_{\mathbf{2 2}}=-1.5 R_{3} .
$$

Proof. Based on the Ref. [46] and (13), we choose the Lyapunov-Krasovskii functional as

$$
V\left(\varepsilon_{t}\right)=\sum_{i=1}^{6} V_{i}\left(\varepsilon_{t}\right), \quad \forall t \in \mathbf{\Xi}_{l}
$$

with

$$
\begin{aligned}
V_{1}\left(\varepsilon_{t}\right)= & v_{1}^{T}(t) P v_{1}(t)+v_{2}^{T}(t)\left(\tau(t) P_{1}\right) v_{2}(t)+v_{3}^{T}(t)\left(\bar{\tau}(t) P_{2}\right) v_{3}(t)+\int_{t-\tau(t)}^{t} v_{4}^{T}(s) Q_{1} v_{4}(s) d s+\int_{t-\bar{\tau}}^{t-\tau(t)} \varepsilon^{T}(s) Q_{2} \varepsilon(s) d s, \\
V_{2}\left(\varepsilon_{t}\right)= & \frac{\alpha^{2}(t)}{2}+\frac{\beta^{2}(t)}{2}+\int_{t-\eta(t)}^{t}\left[\varepsilon^{T}(s) Q_{3} \varepsilon(s)+\dot{\varepsilon}^{T}(s) Q_{4} \dot{\varepsilon}(s)\right] d s+\int_{t-\bar{h}}^{t(t)}\left[\varepsilon^{T}(s) Q_{5} \varepsilon(s)+\dot{\varepsilon}^{T}(s) Q_{6} \dot{\varepsilon}(s)\right] d s \\
& +2 \sum_{i=1}^{n}\left\{q_{i} \int_{0}^{E_{i} \varepsilon(t)}\left[f_{i}(s)-\sigma_{i}^{-} s\right] d s+r_{i} \int_{0}^{E_{i} \varepsilon(t)}\left[\sigma_{i}^{+} s-f_{i}(s)\right] d s\right\}
\end{aligned}
$$


with $V_{i}\left(\varepsilon_{t}\right)(i=3,4,5,6)$ identical to the corresponding ones in $(13)$ and

$$
\begin{aligned}
& v_{1}^{T}(t)=\left[\varepsilon^{T}(t), \varepsilon^{T}(t-\tau(t)), \int_{t-\tau(t)}^{t} \varepsilon^{T}(s) d s, \int_{t-\bar{\tau}}^{t-\tau(t)} \varepsilon^{T}(s) d s\right], \quad v_{2}^{T}(t)=\left[\varepsilon^{T}(t), \varepsilon^{T}(t-\tau(t)), \rho^{T}(t)\right], \\
& v_{3}^{T}(t)=\left[\varepsilon^{T}(t), \varepsilon^{T}(t-\tau(t)), \sigma^{T}(t)\right], \quad v_{4}^{T}(s)=\left[\varepsilon^{T}(s), \dot{\varepsilon}^{T}(s)\right] .
\end{aligned}
$$

Then the derivative of $V_{1}\left(\varepsilon_{t}\right)$ along the system (11) can be directly obtained as

$$
\begin{aligned}
\dot{V}_{1}\left(\varepsilon_{t}\right)= & 2 v_{1}^{T}(t) P \dot{v}_{1}(t)+v_{2}^{T}(t) \dot{\tau}(t) P_{1} v_{2}(t)+2 v_{2}^{T}(t) \tau(t) P_{1} \dot{v}_{2}(t)-v_{3}^{T}(t) \dot{\tau}(t) P_{2} v_{3}(t)+2 v_{3}^{T}(t) \bar{\tau}(t) P_{2} \dot{v}_{3}(t) \\
& +v_{4}^{T}(t) Q_{1} v_{4}(t)-(1-\dot{\tau}(t))\left[v_{4}^{T}(t-\tau(t)) Q_{1} v_{4}(t-\tau(t))-\varepsilon^{T}(t-\tau(t)) Q_{2} \varepsilon(t-\tau(t))\right] \\
& -\varepsilon^{T}(t-\bar{\tau}) Q_{2} \varepsilon(t-\bar{\tau}) \\
= & \varsigma^{T}(t) \operatorname{sym}\left(\bar{\Psi}_{1}(t) P \bar{\Psi}_{2}^{T}(t)+\hat{\Psi}_{1} P_{1} \hat{\Psi}_{2}^{T}(t)+\tilde{\Psi}_{1} P_{2} \tilde{\Psi}_{2}^{T}(t)\right) \varsigma(t)+\varsigma^{T}(t)\left[\hat{\Psi}_{1}\left(\dot{\tau}(t) P_{1}\right) \hat{\Psi}_{1}^{T}-\tilde{\Psi}_{1}\left(\dot{\tau}(t) P_{2}\right) \tilde{\Psi}_{1}^{T}\right. \\
& \left.-(1-\dot{\tau}(t)) \check{\Psi}_{2}\left(Q_{1}-\left[\begin{array}{cc}
Q_{2} & 0 \\
* & 0
\end{array}\right]\right) \check{\Psi}_{2}^{T}\right] \varsigma(t)+\varsigma^{T}(t) \check{\Psi}_{1} Q_{1} \check{\Psi}_{1}^{T} \varsigma(t)-\varepsilon^{T}(t-\bar{\tau}) Q_{2} \varepsilon(t-\bar{\tau})
\end{aligned}
$$

and $\dot{V}_{i}\left(\varepsilon_{t}\right)(i=2,3,4,5,6)$ can be derived as the ones in Theorem 1. Now together with (14)-(32), denotations in (37)-(40), and (44), we can achieve

$$
\begin{aligned}
\dot{V}\left(\varepsilon_{t}\right) \leq & \varsigma^{T}(t)\left\{\boldsymbol{\Omega}+\boldsymbol{\Upsilon}_{1}+\boldsymbol{\Upsilon}_{2}(t)+\eta(t) \mathbf{\Psi}_{1}+\bar{\eta}(t) \mathbf{\Psi}_{2}+\tau(t) \boldsymbol{\Theta}_{1}+\bar{\tau}(t) \boldsymbol{\Theta}_{2}+\frac{\eta(t)}{\bar{h}^{2}} \Gamma_{2}^{T} U_{2} \bar{R}_{2}^{-1} U_{2}^{T} \Gamma_{2}\right. \\
& \left.+\frac{\bar{\eta}(t)}{\bar{h}^{2}} \Gamma_{1}^{T} U_{2} \bar{R}_{2}^{-1} U_{2}^{T} \Gamma_{1}+\frac{\tau(t)}{\bar{\tau}^{2}} \Pi_{2}^{T} U_{1} \bar{R}_{1}^{-1} U_{1}^{T} \Pi_{2}+\frac{\bar{\tau}(t)}{\bar{\tau}^{2}} \Pi_{1}^{T} U_{1} \bar{R}_{1}^{-1} U_{1}^{T} \Pi_{1}\right\} \varsigma(t) \\
\doteq & \varsigma^{T}(t)\left[\mathbf{\Phi}(\tau(t), \eta(t), \dot{\tau}(t))+\frac{\eta(t)}{\bar{h}^{2}} \Gamma_{2}^{T} U_{2} \bar{R}_{2}^{-1} U_{2}^{T} \Gamma_{2}+\frac{\bar{\eta}(t)}{\bar{h}^{2}} \Gamma_{1}^{T} U_{2} \bar{R}_{2}^{-1} U_{2}^{T} \Gamma_{1}+\frac{\tau(t)}{\bar{\tau}^{2}} \Pi_{2}^{T} U_{1} \bar{R}_{1}^{-1} U_{1}^{T} \Pi_{2}\right. \\
& \left.+\frac{\bar{\tau}(t)}{\bar{\tau}^{2}} \Pi_{1}^{T} U_{1} \bar{R}_{1}^{-1} U_{1}^{T} \Pi_{1}\right] \varsigma(t) \doteq \varsigma^{T}(t) \overline{\mathbf{\Phi}}(\tau(t), \eta(t), \dot{\tau}(t)) \varsigma(t),
\end{aligned}
$$

where $\varsigma(t)$ is given in (36) and $\boldsymbol{\Omega}, \boldsymbol{\Upsilon}_{1}, \boldsymbol{\Upsilon}_{2}(t), \mathbf{\Psi}_{1}, \mathbf{\Psi}_{2}, \boldsymbol{\Theta}_{1}, \boldsymbol{\Theta}_{2}$ are expressed in (41)-(42). Especially, $\overline{\mathbf{\Phi}}(\tau(t), \eta(t), \dot{\tau}(t))$ can be rewritten as the following form:

$$
\overline{\mathbf{\Phi}}(\tau(t), \eta(t), \dot{\tau}(t)) \doteq \overline{\boldsymbol{\Phi}}_{1}+\dot{\tau}(t) \overline{\mathbf{\Phi}}_{2}+\tau(t)\left[\overline{\mathbf{\Phi}}_{3}+\dot{\tau}(t) \overline{\mathbf{\Phi}}_{4}\right]+\eta(t) \overline{\mathbf{\Phi}}_{5} .
$$

Then together with the methods in Ref. [18], one can check that if

$$
\begin{aligned}
& \overline{\mathbf{\Phi}}(0,0, \dot{\tau}(t))=\boldsymbol{\Phi}(0,0, \dot{\tau}(t))+\frac{1}{\bar{h}} \Gamma_{1}^{T} U_{2} \bar{R}_{2}^{-1} U_{2}^{T} \Gamma_{1}+\frac{1}{\bar{\tau}} \Pi_{1}^{T} U_{1} \bar{R}_{1}^{-1} U_{1}^{T} \Pi_{1}<0, \\
& \overline{\mathbf{\Phi}}(\bar{\tau}, 0, \dot{\tau}(t))=\boldsymbol{\Phi}(\bar{\tau}, 0, \dot{\tau}(t))+\frac{1}{\bar{h}} \Gamma_{1}^{T} U_{2} \bar{R}_{2}^{-1} U_{2}^{T} \Gamma_{1}+\frac{1}{\bar{\tau}} \Pi_{2}^{T} U_{1} \bar{R}_{1}^{-1} U_{1}^{T} \Pi_{2}<0, \\
& \overline{\mathbf{\Phi}}(0, \bar{h}, \dot{\tau}(t))=\boldsymbol{\Phi}(0, \bar{h}, \dot{\tau}(t))+\frac{1}{\bar{h}} \Gamma_{2}^{T} U_{2} \bar{R}_{2}^{-1} U_{2}^{T} \Gamma_{2}+\frac{1}{\bar{\tau}} \Pi_{1}^{T} U_{1} \bar{R}_{1}^{-1} U_{1}^{T} \Pi_{1}<0, \\
& \overline{\mathbf{\Phi}}(\bar{\tau}, \bar{h}, \dot{\tau}(t))=\boldsymbol{\Phi}(\bar{\tau}, \bar{h}, \dot{\tau}(t))+\frac{1}{\bar{h}} \Gamma_{2}^{T} U_{2} \bar{R}_{2}^{-1} U_{2}^{T} \Gamma_{2}+\frac{1}{\bar{\tau}} \Pi_{2}^{T} U_{1} \bar{R}_{1}^{-1} U_{1}^{T} \Pi_{2}<0,
\end{aligned}
$$

then $\overline{\mathbf{\Phi}}(\tau(t), \eta(t), \dot{\tau}(t))<0$ can hold. Now together with Schur-complement and convex combination technique, one can check that the terms in (41)-(42) guarantee (47)-(50) to be true, which means $\overline{\mathbf{\Phi}}(\tau(t), \eta(t), \dot{\tau}(t))<0$. Thus once the conditions in (41)-(42) hold, the systems (1)-(2) achieve the desired synchronization.

Remark 4. During proving Theorems 1-2, the LKF terms in $V\left(\varepsilon_{t}\right)$ have efficiently utilized the information on $\tau(t), \eta(t)$, nonlinear function $f(E \varepsilon(t))$, and adaptive conditions $\alpha(t), \beta(t)$, some of which have not been considered 
in present works. Especially, two improved discontinuous Lyapunov terms and delay-product-type ones have been put forward to reduce the conservatism. Though the derived LMI-based conditions are presented in terms of complicated forms and induce the computational complexity, it is convenient to check their feasibility without tuning any parameters by resorting to the most recently developed algorithms.

Remark 5. On the basis of the discussions in $[9,21,22,41]$, these integral inequalities described by Lemmas $1-4$ can can be more efficient than ever. Therefore, together with these integral inequalities and improved ones, based on the multiple integral LKF terms in (13) and (43), our work has effectively tackled the issue on delay-dependence and the previously ignored information has been fully studied. Furthermore, since two types of time-varying delays were involved in the systems, some novel Lyapunov terms have been presented to deal with their interconnection, which could play an important role in reducing the conservatism $[8,24,37,39]$.

Remark 6. Most recently, the Refs. [12,30,46-48] put forward some novel techniques to reduce the conservatism. In [12], the novelty relies on second-order Bessel-Legendre integral inequality which is less conservative than the Wirtinger-based ones. Later, the Ref. [30] extends the methods in [12] to arbitrary orders, which can further reduce the conservatism. The Ref. [46] presents some novel delay-product-type LKFs that is tightly related to integral inequalities. The work [47] combines the Wirtinger-based inequality (or Auxiliary function-based inequality) and reciprocally convex technique to achieve a set of novel integral inequalities, which later receive some improvement [48]. In this work, we have resorted to the methods in $[46,48]$ to obtain the theorems.

Remark 7. The methods of this work can be extended to many cases. Firstly, as for uncertainties and time-delay, distributed complex-valued parameters [31], polytopic uncertain ones and norm-bounded uncertain ones [10], nonlinear uncertain ones [11], and distributed time-delay [10] can be added into the systems (1)-(2) and the corresponding results can be obtained. Secondly, as illustrated in Refs. [30,34], sensor faults usually occur during sampling data, which can be studied in this work, either. As for the issue, we can design a fault-tolerant controller in the presence of actuator faults and loss of effectiveness of the actuator. Furthermore, in order to tackle the fault failures, the adaptive event-triggered schemes also need some improvements.

Remark 8. Overall, the theoretic contributions of this work can be elaborated as: firstly, a novel adaptive eventtriggered scheme was presented, which can wholly reflect the real-time information of controlled systems; secondly, in $V_{3}\left(\varepsilon_{t}\right)$, two triple Lyapunov terms were employed to reduce the conservatism; thirdly, in $V_{4}\left(\varepsilon_{t}\right)$, some novel LKF terms were put forward to represent the interconnection between $\tau(t)$ and $\eta(t)$, which has not appeared in present works; fourthly, as for those recently reported LKF terms in $[13,16,18,46]$, our work have combined and improved them to extend the application areas.

\section{Numerical examples}

In this section, two numerical examples with comparisons will be presented to illustrate the derived results.

Example 1. As a special case of systems (1)-(2), we will use the following Chua's circuit to illustrate our master-slave synchronization results

$$
\left\{\begin{array}{l}
\dot{x}_{1}=\alpha\left[-m_{1} x_{1}+x_{2}+h\left(x_{1}\right)\right] \\
\dot{x}_{2}=x_{1}-x_{2}+x_{3} \\
\dot{x}_{3}=-\beta x_{2}-x_{3}
\end{array}\right.
$$

with $h\left(x_{1}\right)=\frac{1}{2}\left(m_{1}-m_{0}\right)\left(\left|x_{1}+c\right|-\left|x_{1}-c\right|\right)$ and the parameters expressed as $m_{0}=-\frac{1}{7}, m_{1}=\frac{2}{7}, \alpha=9, \beta=$ $14.286, c=1$. Then the system can be represented in the form of Lur'e model (1) with

$$
A=\left[\begin{array}{ccc}
-\frac{18}{7} & 9 & 0 \\
1 & -1 & 1 \\
0 & -14.286 & 0
\end{array}\right], B=\left[\begin{array}{ccc}
0 & 0 & 0 \\
0 & 0 & 0 \\
0 & 0 & 0
\end{array}\right], \quad D=\left[\begin{array}{ccc}
\frac{27}{7} & 0 & 0 \\
0 & 0 & 0 \\
0 & 0 & 0
\end{array}\right], E=\left[\begin{array}{ccc}
1 & 0 & 0 \\
0 & 0 & 0 \\
0 & 0 & 0
\end{array}\right], \quad F=\left[\begin{array}{lll}
1 & 0 & 0
\end{array}\right],
$$

and $f_{1}\left(x_{1}\right)=\frac{1}{2}\left(\left|x_{1}+1\right|-\left|x_{1}-1\right|\right)$ belonging to the sector $[0, k]$ with $k=1$. Now, based on the adaptive eventtriggered scheme (AETS) in (7)-(9) and the controller in (10), if we set $\bar{d}=0, \underline{h}=\bar{h}=0.3, \bar{\tau}=0.01, \mu_{0}=\mu_{1}=0$, 
and $\delta=0.2, \delta_{1}=\delta_{2}=5, \theta_{1}=\theta_{2}=2$, then by resorting to LMI in Matlab Toolbox, one can check that the feasible solution to the LMIs in Theorem 1 is existent. Especially, the triggering parameters $\Phi_{i}(i=1,2)$ and the controller gain $K$ can be respectively computed out as

$$
\Phi_{1}=\left[\begin{array}{ccc}
0.8638 & 0.1420 & -0.2641 \\
0.1420 & 0.8234 & -0.1123 \\
-0.2641 & -0.1123 & 1.0464
\end{array}\right], \Phi_{2}=\left[\begin{array}{ccc}
0.7435 & -0.2537 & 0.3492 \\
-0.2537 & 0.9036 & 0.2502 \\
0.3492 & 0.2502 & 0.9733
\end{array}\right], K=\left[\begin{array}{c}
3.0238 \\
0.1684 \\
-2.7955
\end{array}\right] .
$$

Furthermore, if we set $\alpha=\beta=0.2$, then the scheme in (7) can be converted into the traditional event-triggered scheme (TETS). Now together with Theorem 1 and Remark 3, we also can derive the corresponding triggering parameters $\Phi_{i}(i=1,2)$ and the controller gain $K$ with $\bar{d}=0, \underline{h}=\bar{h}=0.3, \bar{\tau}=0.01, \mu_{0}=\mu_{1}=0$. By denoting $z=\left[\begin{array}{lll}z_{1} & z_{2} & z_{3}\end{array}\right]^{T}, x=\left[\begin{array}{lll}x_{1} & x_{2} & x_{3}\end{array}\right]^{T}$, and $\varepsilon=z-x=\left[\begin{array}{lll}z_{1}-x_{1} & z_{2}-x_{2} & z_{3}-x_{3}\end{array}\right]^{T}$, then the state trajectories of $z(t), x(t)$, and the error state $\varepsilon(t)$ can be respectively presented in Fig. 1 with the initial conditions $x(0)=[-1,0.8,-0.3]^{T}$ and $z(0)=[0.8,-0.3,0.7]^{T}$. The transmission instants and releasing intervals by AETS and TETS can be illustrated in Fig. 2. One can check that the AETS releases less transmitted data than the ones by TETS. Especially, by setting $\alpha(0)=0.648, \beta(0)=0.852$ guaranteeing $0.2 \leq \alpha(0), \beta(0) \leq 2$, we also present the tendency of the functions $\alpha(t), \beta(t)$ in Fig. 3. Then from Fig. 3, one check that once the desired synchronization is achieved, the functions $\alpha(t), \beta(t)$ can converge to be constants with time on.

Meanwhile, since this example has been widely studied, thus we aim to give some comparisons on maximum allowable upper bounds (MAUBs) of $\bar{h}$ with the ones in the Refs. $[8,13,14,25,39]$. By setting $\bar{d}=0, \underline{h}=0.1, \bar{\tau}=0.01, \mu_{0}=$ $\mu_{1}=0$ in Theorem 1 and Theorem 2, the MAUBs of $\bar{h}$ can be computed out and listed in Table 1 . To show the superiorities of our methods, we still use the theorems in $[8,13,14,25,39]$ to obtain the corresponding MAUBs and controller gains, which can be illustrated in Table 1. From Table 1, it is clear that our results present much less conservatism than some existing ones. Especially, one can check that the MAUB by Theorem 2 is larger than the one by Theorem 1, which means that the delay-product-type LKF terms can reduce the conservatism more effectively. Especially, the numbers of decision variables (NoDVs) are also presented in Table 1.

Table 1

The corresponding MAUBs of $\bar{h}$ for various methods and NoDVs in Example 1.

\begin{tabular}{|c|c|c|c|}
\hline Utilized methods & MAUBs on $\bar{h}$ & Controller gain $K$ & NoDVs \\
\hline Theorem $3.1[8]$ & 0.4355 & {$\left[\begin{array}{lll}3.8449 & 0.3853 & -3.4730\end{array}\right]^{T}$} & $14.5 n^{2}+6.5 n$ \\
\hline Theorem $3.1[25]$ & 0.4687 & {$\left[\begin{array}{lll}3.7455 & 0.2457 & -2.5735\end{array}\right]^{T}$} & $21.5 n^{2}+12.5 n$ \\
\hline Theorem $3.1[14]$ & 0.5000 & {$\left[\begin{array}{lll}6.7420 & 1.7730 & -5.9268\end{array}\right]^{T}$} & $13.5 n^{2}+4.5 n$ \\
\hline Theorem 1 [39] & 0.5185 & {$\left[\begin{array}{lll}3.1756 & 0.1406 & -2.8992\end{array}\right]^{T}$} & $15.5 n^{2}+8.5 n$ \\
\hline Theorem $2[13]$ & 0.5386 & {$\left[\begin{array}{lll}3.1152 & 0.1746 & -2.7955\end{array}\right]^{T}$} & $47.5 n^{2}+7.5 n$ \\
\hline Theorem 1 & 0.5584 & {$\left[\begin{array}{lll}3.0225 & 0.2087 & -3.2440\end{array}\right]^{T}$} & $27.5 n^{2}+15.5 n$ \\
\hline Theorem 2 & 0.5775 & {$\left[\begin{array}{lll}3.0124 & 0.1756 & -3.2254\end{array}\right]^{T}$} & $37.5 n^{2}+19.5 n$ \\
\hline
\end{tabular}

Example 2. Together with the state delay $\tau(t)$, we consider the master system (1) and the slave one (2) with

$$
A=\left[\begin{array}{cc}
-0.7 & 0.4 \\
0.6 & -0.8
\end{array}\right], B=\left[\begin{array}{cc}
0.1 & -0.05 \\
0.05 & 0.1
\end{array}\right], D=\left[\begin{array}{cc}
2.1 & -0.2 \\
-5.0 & 3.0
\end{array}\right], E=F=\left[\begin{array}{ll}
1 & 0 \\
0 & 1
\end{array}\right]
$$

and the nonlinear functions can be selected as $f_{i}(s)=\tanh (s)(i=1,2)$. Firstly, in Table 2 , we will give some comparing results on MAUBs of $\bar{h}$ with the Refs. [24,37,39]. Similar to Example 1 , we still utilize Theorem 1 and Theorem 2 to check the superiorities. Prior to solving the derived LMIs, since time-delay involved in $[24,37,39]$ are constant ones, the condition $\mu_{0}=\mu_{1}=0$ has to imposed. During the computing, we will set $\bar{d}=0, \underline{h}=0.1, \bar{\tau}=$ $0.45, \delta=0.2, \delta_{1}=\delta_{2}=6, \theta_{1}=\theta_{2}=2$ in Theorem 1 and Theorem 2 . Then the corresponding MAUBs can be listed in Table 2. Secondly, in Table 3, we will set $\bar{h}=0.35$ and give some comparing results on the MAUBs of $\bar{\tau}$ with the Refs. [24,37,39], which can help check the superiorities of delay-product-type LKF technique. 

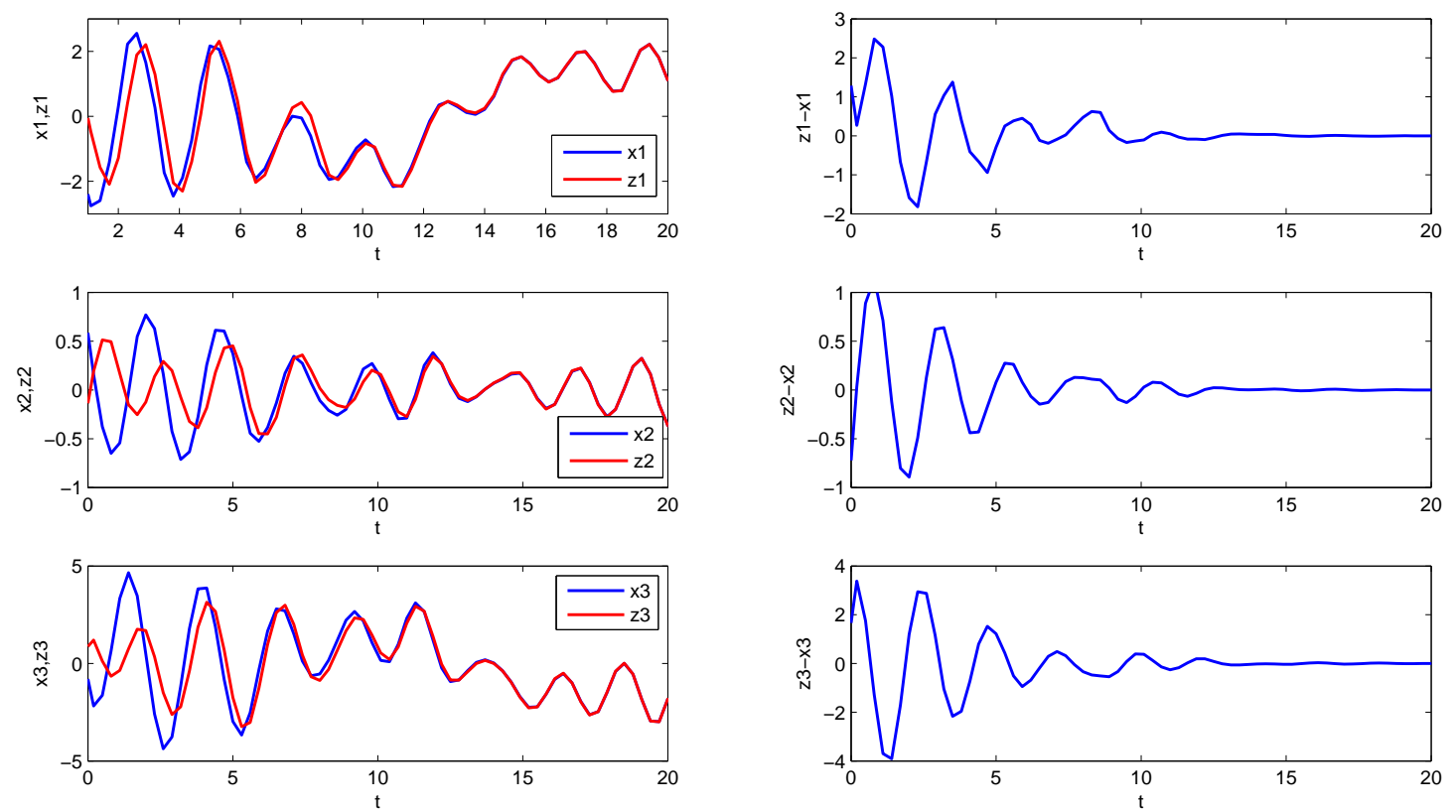

Fig. 1. State trajectories of the master system, slave system, and error one.
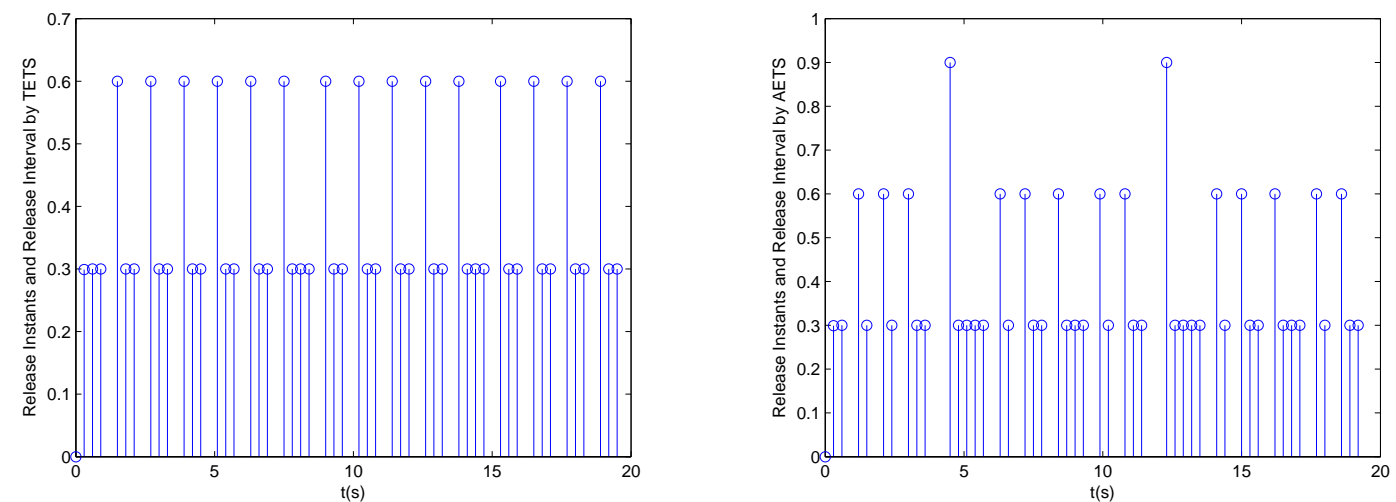

Fig. 2. Transmission instants and releasing intervals of TETS (left) and AETS (right).

Table 2

The corresponding MAUBs of $\bar{h}$ for various methods and $\bar{\tau}=0.45$ in Example 2 .

\begin{tabular}{cccccc}
\hline Methods & Theorem 2 [37] & Theorem 1 [39] & Theorem 1 [24] & Theorem 1 & Theorem 2 \\
\hline $\bar{h}$ & 0.2975 & 0.3824 & 0.3537 & 0.4125 & 0.4253 \\
\hline
\end{tabular}

Table 3

The corresponding MAUBs of $\bar{\tau}$ for various methods and $\bar{h}=0.35$ in Example 2 .

\begin{tabular}{cccccc}
\hline Methods & Theorem 2 [37] & Theorem 1 [39] & Theorem 1 [24] & Theorem 1 & Theorem 2 \\
\hline $\bar{\tau}$ & 0.3372 & 0.4635 & 0.4527 & 0.4790 & 0.5025 \\
\hline
\end{tabular}

It follows from the tables above that our results can be more efficient than the ones in $[24,37,39]$. Furthermore, our results can tackle time-varying delay while the ones in $[24,37,39]$ fail to verify this case. 

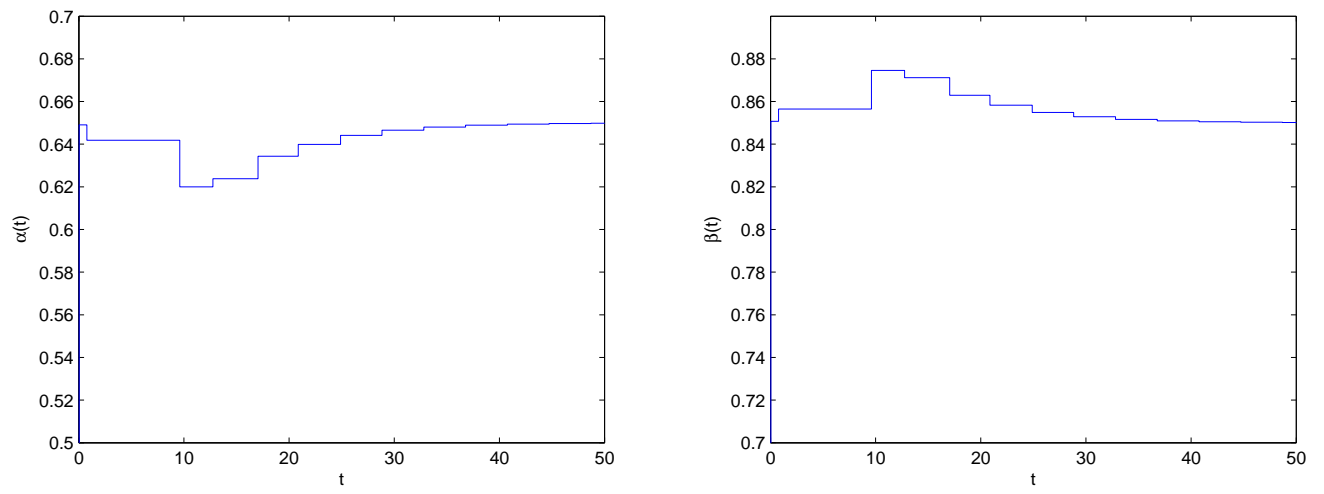

Fig. 3. The trajectories of the event adaptive laws $\alpha(t), \beta(t)$.

\section{Conclusion}

In the paper, the problem on designing the adaptive event-triggering scheme and sampled-data controller of masterslave synchronization has been studied for master-slave Lur'e systems with time-varying delay. In order to reduce the conservatism, some novel Wirtinger-based inequalities and two augmented LKFs have been put forward during the discussions. Together with stability criteria on synchronization error system, two sufficient conditions have been obtained and the controller gains can be checked by solving the derived LMIs. Finally, two numerical examples have demonstrated that our results could produce less conservatism than some reported ones.

\section{Acknowledgements}

This work is supported by National Natural Science Foundation of China (Nos. 61473079, 61473115), Jiangsu Natural Science Foundation (Nos. BK20171419, BK20150888), Natural Science Foundation for Jiangsu's Universities (No. 15KJB12004), and Fundamental Research Fund for Central Universities (Nos. NS2016030, NJ20160024).

\section{A Appendix}

$$
\begin{aligned}
& \bar{\tau}(t)=\bar{\tau}-\tau(t), \quad \bar{\eta}(t)=\bar{h}-\eta(t), \quad \bar{\mu}=\mu_{1}-\mu_{0}, \quad \bar{\theta}=\bar{\tau}-\bar{h}, \quad \bar{\delta}=\bar{\tau}^{2}-\bar{h}^{2} ; \\
& \rho(t)=\frac{1}{\tau(t)} \int_{t-\tau(t)}^{t} \varepsilon(s) d s, \quad \sigma(t)=\frac{1}{\bar{\tau}(t)} \int_{t-\bar{\tau}}^{t-\tau(t)} \varepsilon(s) d s, \varrho(t)=\frac{1}{\eta(t)} \int_{t-\eta(t)}^{t} \varepsilon(s) d s, \vartheta(t)=\frac{1}{\bar{\eta}(t)} \int_{t-\bar{h}}^{t-\eta(t)} \varepsilon(s) d s ; \\
& \nu(t)=\frac{2}{\tau^{2}(t)} \int_{t-\tau(t)}^{t} \int_{t-\tau(t)}^{s} \varepsilon(u) d u d s, \omega(t)=\frac{2}{\bar{\tau}^{2}(t)} \int_{t-\bar{\tau}}^{t-\tau(t)} \int_{t-\bar{\tau}}^{s} \varepsilon(u) d u d s, \psi(t)=\frac{1}{\bar{\theta}} \int_{t-\bar{\tau}}^{t-\bar{h}} \varepsilon(s) d s ; \\
& \phi(t)=\frac{2}{\eta^{2}(t)} \int_{t-\eta(t)}^{t} \int_{t-\eta(t)}^{s} \varepsilon(u) d u d s, \varphi(t)=\frac{2}{\bar{\eta}^{2}(t)} \int_{t-\bar{h}}^{t-\eta(t)} \int_{t-\bar{h}}^{s} \varepsilon(u) d u d s, \chi(t)=\frac{2}{\bar{\theta}^{2}} \int_{t-\bar{\tau}}^{t-\bar{h}} \int_{t-\bar{\tau}}^{s} \varepsilon(u) d u d s ; \\
& \xi^{T}(t)=\left[\begin{array}{lllllllllllllll}
\varepsilon^{T}(t) & \varepsilon^{T}(t-\tau(t)) & \varepsilon^{T}(t-\bar{\tau}) & \varepsilon^{T}\left(i_{l}\right) & \varepsilon^{T}(t-\bar{h}) & f^{T}(E \varepsilon(t)) & \rho^{T}(t) & \sigma^{T}(t) & \varrho^{T}(t) & \theta^{T}(t) & \nu^{T}(t)
\end{array}\right. \\
& \left.\begin{array}{llllllll}
\omega^{T}(t) & \phi^{T}(t) & \varphi^{T}(t) & \psi^{T}(t) & \chi^{T}(t) & \dot{\varepsilon}^{T}(t) & e^{T}\left(i_{l}\right) & \dot{\varepsilon}^{T}(t-\bar{h})
\end{array}\right] \\
& e_{i}=\left[\begin{array}{lll}
0_{n \times(i-1) n} & I_{n} & 0_{n \times(19-i) n}
\end{array}\right]^{T} \quad(1 \leq i \leq 19) ; \\
& \Upsilon_{0}=\left[\begin{array}{lll}
e_{1} & e_{5} & 0_{19 n \cdot n}
\end{array}\right], \Upsilon_{1}=\left[\begin{array}{ll}
0_{19 n \cdot 2 n} & e_{9}
\end{array}\right], \Upsilon_{2}=\left[\begin{array}{ll}
0_{19 n \cdot 2 n} & e_{10}
\end{array}\right], \Lambda=\left[\begin{array}{lll}
e_{1} & e_{5} & 0_{19 n \cdot 2 n}
\end{array}\right] \text {; }
\end{aligned}
$$




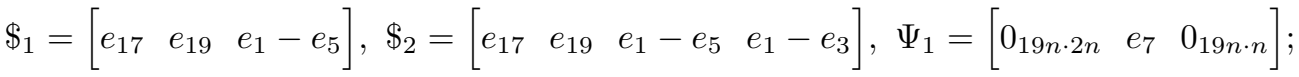

$$
\begin{aligned}
& \Psi_{2}=\left[\begin{array}{lll}
0_{19 n \cdot 2 n} & e_{8} & 0_{19 n \cdot n}
\end{array}\right], \Phi_{1}=\left[\begin{array}{ll}
0_{19 n \cdot 3 n} & e_{9}
\end{array}\right], \Phi_{2}=\left[\begin{array}{ll}
0_{19 n \cdot 3 n} & e_{10}
\end{array}\right] \\
& \Pi_{1}=\left[\begin{array}{c}
e_{1}-e_{2} \\
e_{1}+e_{2}-2 e_{7} \\
e_{1}-e_{2}+6 e_{7}-12 e_{11}
\end{array}\right], \Pi_{2}=\left[\begin{array}{c}
e_{2}-e_{3} \\
e_{2}+e_{3}-2 e_{8} \\
e_{2}-e_{3}+6 e_{8}-12 e_{12}
\end{array}\right] ; \\
& \Gamma_{1}=\left[\begin{array}{c}
e_{1}-e_{4} \\
e_{1}+e_{4}-2 e_{9} \\
e_{1}-e_{4}+6 e_{9}-12 e_{13}
\end{array}\right], \Gamma_{2}=\left[\begin{array}{c}
e_{4}-e_{5} \\
e_{4}+e_{5}-2 e_{10} \\
e_{4}-e_{5}+6 e_{10}-12 e_{14}
\end{array}\right]
\end{aligned}
$$

\section{References}

[1] T. Carroll, L. Pecora, Synchronization chaotic circuits, IEEE Transactions on Circuits and Systems-I 38 (1991) $453-56$.

[2] J.D. Cao, R. Sivasamy, R. Rakkiyappan, Sampled-data $H_{\infty}$ synchronization of chaotic Lur'e systems with time delay, Circuits Systems \& Signal Processing 35 (2016) 811-835.

[3] W. Chen, D. Wei, X. Lu, Global exponential synchronization of nonlinear time-delay Lur'e systems via delayed impulsive control, Communications in Nonlinear Science and Numerical Simulation 3 (2014) 3298-3312.

[4] J. Dai, G. Guo, Event-based consensus for second-order multi-agent systems with actuator saturation under fixed and Markovian switching topologies, Journal of the Franklin Institute 354 (2017) 6098-6118.

[5] Y.G. Chen, W. Qian, S.M. Fei, Improved robust stability conditions for uncertain neutral systems with discrete and distributed delays, Journal of the Franklin Institute 6 (2015) 2634-2645.

[6] M. Fischmann, J. Flores, J. Gomes, Dynamic controller design for synchronization of Lur'e type systems subject to control saturation, IFAC-PapersOnLine 50 (2017) 11853-11858.

[7] Z. Gu, L. Yang, E.G. Tian, H. Zhao, Event-triggered reliable $H_{\infty}$ filter design for networked systems with multiple sensor distortions: a probabilistic partition approach, ISA Transactions 66 (2017) 2-9.

[8] C.C. Hua, C. Ge, X.P. Guan, Synchronization of chaotic Lur'e systems with time delays using sampled-data control, IEEE Transactions on Neural Networks and Learning Systems 26 (2015) 1214-1221.

[9] C.C. Hua, S. Wu, X. Yang, X.P. Guan, Stability analysis of time-delay systems via free-matrix-based double integral inequality, International Journal of Systems Science 48 (2017) 257-263.

[10] H. Karimi, H. Gao, New delay-dependent exponential $H_{\infty}$ synchronization for uncertain neural networks with mixed time delays, IEEE Transactions on Systems, Man, and Cybernetics-Part B, 40(2010) 173- 185.

[11] H. Karimi, A sliding mode approach to $H_{\infty}$ synchronization of master-slave time-delay systems with Markovian jumping parameters and nonlinear uncertainties, Journal of the Franklin Institute, 349(2012) 1480-1496.

[12] K. Liu, A. Seuret, Y.Q. Xia, Stability analysis of systems with time-varying delays via the second-order Bessel-Legendre inequality, Automatica 76(2017) 138-142.

[13] T. Lee, J.H. Park, Improved criteria for sampled-data synchronization of chaotic Lur'e systems using two new approaches, Nonlinear Analysis: Hybrid Systems 24 (2017) 132-145.

[14] S. Liu, L. Zhou, Network synchronization and application of chaotic Lur'e systems based on event-triggered mechanism, Nonlinear Dynamics 83 (2016) 2497-2507.

[15] T. Lee, J.H. Park, Stability analysis of sampled-data systems via free-matrix-based time-dependent discontinuous Lyapunov approach, IEEE Transactions on Automatic Control 62 (2017) 3653-3657.

[16] K. Liu, E. Fridman, Wirtinger's inequality and Lyapunov-based sampled-data stabilization, Automatica 48 (2012) $102{ }^{*} \mathrm{C} 108$.

[17] T. Li, T. Wang, A.G. Song, S.M. Fei, Delay-derivative-dependent stability for delayed neural networks with unbounded distributed delay, IEEE Transactions on Neural Networks 21 (2010) 1365-1371.

[18] F. Long, C. K. Zhang, Y. He, L. Jiang, M. Wu, Stability analysis of Lur'e systems with additive delay components via a relaxed matrix inequality, Applied Mathematics and Computation, 328 (2018) 224-242.

[19] J. Mao, J. Guo, Z.R. Xiang, Sampled-data control of a class of uncertain switched nonlinear systems in nonstrict-feedback form, International Journal of Robust \& Nonlinear Control (2017) DOI: 10.1002/rnc.39075.

[20] L. Pecora, T. Carroll, Synchronization in chaotic systems, Physical Review Letters 64 (1990) 821-824 .

[21] M. Park, O. Kwon, J. Park, S. Lee, Stability of time-delay systems via Wirtinger-based double integral inequality, Automatica 55 (2015) 204-208.

[22] P. Park, W. Lee, S. Lee. Auxiliary function-based integral inequalities for quadratic functions and their applications to time-delay systems, Journal of the Franklin Institute 352 (2016) 1378-1396. 
[23] K.B. Shi, X.Z. Liu, H. Zhu, M.S. Zhong, C. Yin, Novel delay-dependent master-slave synchronization criteria of chaotic Lur'e systems with time-varying-delay feedback control, Applied Mathematics and Computation 282 (2016) 137-154.

[24] K.B. Shi, X.Z. Liu, H. Zhu, M.S. Zhong, Y. Liu, Novel integral inequality approach on master-slave synchronization of chaotic delayed Lur'e systems with sampled-data feedback control, Nonlinear Dynamics 83 (2016) 1259-1274.

[25] K.B. Shi, X.Z. Liu, H. Zhu, M.S. Zhong, On designing stochastic sampled-data controller for master-slave synchronization of chaotic Lur'e system via a novel integral inequality, Communications in Nonlinear Science and Numerical Simulation 34 (2016) $165-184$.

[26] K.B. Shi, Y.Y. Tang, X.Z. Liu, S.M. Zhong, Non-fragile sampled-data robust synchronization of uncertain delayed chaotic Lurie systems with randomly occurring controller gain fluctuation, ISA Transactions 66 (2017) 185-199.

[27] K.B. Shi, X.Z. Liu, Y.Y. Tang, H. Zhu, S.M. Zhong, Some novel approaches on state estimation of delayed neural networks, Information Sciences 372 (2016) 313-331.

[28] S. Senan, M. Syed Ali, R. Vadivel, S. Arik, Decentralized event-triggered synchronization of uncertain Markovian jumping neutraltype neural networks with mixed delays, Neural Networks 86 (2017) 32-41.

[29] A. Seuret, F. Gouaisbaut, Stability of linear systems with time-varying delays using Bessel-Legendre inequalities, IEEE Transactions on Automatic Control 63 (2018) 225-232.

[30] R. Sakthivel, H. Karimi, M. Joby, S. Santra, Resilient sampled-data control for Markovian jump systems with an adaptive faulttolerant mechanism, IEEE Transactions on Circuits and Systems-II, 64 (2017) 1312-1316.

[31] Z. Tai, X. Wang, Y. Shi, H. Karimi, Input-to-state stability of Lur'e hyperbolic distributed complex-valued parameter control systems: LOI approach, Mathematical Problems in Engineering, DOI: 10.1155/2013/364057.

[32] G.H. Wen, G.R. Chen, Z. Chen, X. Yu. Event-triggered master-slave synchronization with sampled-data communication, IEEE Transactions on Circuits and Systems-II 63 (2016) 304-308.

[33] S. Wen, G. Guo, W. Wong, Hybrid event-time-triggered networked control systems: Scheduling-event-control co-design, Information Sciences 305 (2015) 269-284.

[34] Y. Wei, J. Qiu, H. Karimi, Reliable output feedback control of discrete-time fuzzy affine systems with actuator faults, IEEE Transactions on Circuits and Systems-I, 64 (2017) 170-181.

[35] D. Yue, E.G. Tian, Q.L. Han, A delay system method for designing event-triggered controllers of networked control systems, IEEE Transactions on Automatic Control 58 (2013) 475-481.

[36] F. Zhang, H. Trentelman, J. Scherpen, Dynamic feedback synchronization of Lur'e networks via incremental sector doundedness, IEEE Transactions on Automatic Control 61 (2016) 2579-2584.

[37] H. Zeng, H. Ju, S. Xiao, Y. Liu, Further results on sampled-data control for master-slave synchronization of chaotic Lur'e systems with time delay, Nonlinear Dynamics 82 (2015) 1-13.

[38] C.K. Zhang, L. Jiang, Y. He, Q. Wu, Asymptotical synchronization for chaotic Lur'e systems using sampled-data control, Communications in Nonlinear Science and Numerical Simulation 18 (2013) 2743-2751.

[39] R. Zhang, D. Zeng, S. Zhong, Novel master-slave synchronization criteria of chaotic Lur'e systems with time delays using sampleddata control, Journal of the Franklin Institute 354 (2017) 4930-4954.

[40] J. Zhang, C. Peng, Synchronization of master-slave neural networks with a decentralized event triggered communication scheme, Neurocomputing 173 (2010) 1824-1831.

[41] X.M. Zhang, Q.L. Han, A. Seuret, F. Gouaisbaut, An improved reciprocally convex inequality and an augmented Lyapunov-Krasovskii functional for stability of linear systems with time-varying delay, Automatica 84 (2017) 221-226.

[42] X.M. Zhang, Q.L. Han, B. Zhang, An overview and deep investigation on sampled-data-based event-triggered control and filtering for networked systems, IEEE Transactions on Industrial Informatics 12 (2017) 4-16.

[43] X.M. Zhang, Q.L. Han, X.H. Yu, Survey on recent advances in networked control systems, IEEE Transactions on Industrial Informatics 12 (2016) 1740-1752.

[44] X.M. Zhang, Q.L. Han,Event-based $H_{\infty}$ filtering for sampled-data systems, Automatica 51 (2015) 55-69.

[45] W.C. Zou, Z.R. Xiang, Event-triggered distributed containment control of heterogeneous linear multi-agent systems by an output regulation approach, International Journal of Systems Science 48 (2017) 2041-2054.

[46] C.K. Zhang, Y. He, L. Jiang, M. Wu, Notes on stability of time-delay systems: bounding inequalities and augmented LyapunovKrasovskii functionals, IEEE Transactions on Automatic Control, 62 (2017) 5331-5336.

[47] C.K. Zhang, Y. He, L. Jiang, M. Wu, H. Zeng, Stability analysis of systems with time-varying delay via relaxed integral inequalities, Systems \& Control Letters, 92 (2016) 52-61.

[48] C.K. Zhang, Y. He, L. Jiang, M. Wu, Q. Wang, An extended reciprocally convex matrix inequality for stability analysis of systems with time-varying delay, Automatica, 85 (2017) 481-485.

[49] C.K. Zhang, Y. He, L. Jiang, M. Wu, H. Zeng, Stability analysis for delayed neural networks considering both conservativeness and complexity, IEEE Transactions on Neural Networks and Learning Systems, 27 (2016) 1486-1501. 